\title{
FMH-Ärztestatistik 2006
}

\section{Esther Kraft}

Generalsekretariat der Verbindung der Schweizer Ärztinnen und Ärzte FMH
Korrespondenz: Esther Kraft

FMH

Abteilung DDQ Elfenstrasse 18 CH-3000 Bern 15 Tel. 0313591111 Fax 0313591112

esther.kraft@fmh.ch
Die FMH-Ärztestatistik ist eine der wichtigsten Datenquellen zur Beantwortung von Fragen zur Ärztedemographie in der Schweiz. Sie wurde bisher von Jürg Jau, Leiter des Bereichs ICT-Planung und -Konzeption, betreut und basiert auf den Datenerhebungen der Abteilung Dienstleistungen Mitgliedschaft DLM sowie dem Sekretariat Aus-, Weiter- und Fortbildung AWF. Thematisch gehört die Ärztestatistik aber in den Aufgabenbereich der neuen Abteilung Daten, Demographie und Qualität DDQ, die nun auch über zusätzliche personelle Ressourcen verfügt. Deshalb wurde per Ende 2006 die organisatorische Verantwortung an die Abteilung DDQ übertragen. Jürg Jau, dessen kompetente Arbeit wir an dieser Stelle herzlich verdanken, wird der Abteilung DDQ weiterhin beratend zur Verfügung stehen.

Die bisherigen Auswertungen und Analysen sind von einer guten und kontinuierlichen Qualität. Die Abteilung DDQ wird diese Arbeit weiterführen sowie einzelne Bereiche vertiefen. Der FMH-Zentralvorstand hat die dazu benötigten Ressourcen freigegeben und einem Projekt Revision der Ärztestatistik zugestimmt. Dieses Projekt soll die Voraussetzungen schaffen, damit die
Ärztestatistik auch in Zukunft relevante Grundlagen für aktuelle ärztedemographische Diskussionen liefern kann. Zusätzlich soll eine weitere Verbesserung der Datenqualität angestrebt werden. Die aktuellen Definitionen und Begrifflichkeiten werden unter Miteinbezug von Experten und verschiedenen Fachgruppen überprüft und an die aktuellen Gegebenheiten angepasst. Beispielsweise hat sich gezeigt, dass der Begriff der Grundversorger unter Berücksichtigung der Anliegen der Pädiater präziser gefasst werden muss. Da verschiedene Organisationen mit den Zahlen der FMH arbeiten, ist es wichtig, dass die allgemeine Gültigkeit und Akzeptanz gegenüber den Aussagen der Ärztestatistik sowie den zugrunde liegenden Definitionen weiterbesteht.

Die Revision ist in zwei Etappen vorgesehen und wird bei den Publikationen der Ärztestatistiken 2007 (März 2008) und 2008 (März 2009) erstmals wirksam.

Die Tabellen der FMH-Ärztestatistik sind im Internet unter www.fmh.ch abrufbar.

Zur besseren Lesbarkeit der Statistik wird in der Regel der Begriff «Ärzte» anstelle von «Ärztinnen und Ärzten» verwendet.

\section{Allgemeine Übersicht 2006 / Aperçu général 2006}

\begin{tabular}{|c|c|c|c|c|c|c|c|c|c|}
\hline \multirow{2}{*}{$\begin{array}{l}\text { Ärzte in der Schweiz / } \\
\text { Médecins en Suisse }\end{array}$} & \multicolumn{3}{|c|}{ Frauen / Femmes } & \multicolumn{3}{|c|}{ Männer / Hommes } & \multicolumn{3}{|c|}{ Total / Total } \\
\hline & 2006 & 2005 & $\begin{array}{l}\text { Veränd. } / \\
\text { Variation }\end{array}$ & 2006 & 2005 & \begin{tabular}{|l|} 
Veränd.$/$ \\
Variation
\end{tabular} & 2006 & 2005 & $\begin{array}{l}\text { Veränd./ } \\
\text { Variation }\end{array}$ \\
\hline Mit Praxistätigkeit / En pratique privée & 3'926 & 3'776 & $+4.0 \%$ & $11 ' 606$ & $11^{\prime} 537$ & $+0.6 \%$ & $15^{\prime} 532$ & $15^{\prime} 313$ & $+1.4 \%$ \\
\hline in $\% /$ en $\%$ & $25.3 \%$ & $24.7 \%$ & & $74.7 \%$ & $75.3 \%$ & & & & \\
\hline $\begin{array}{r}\text { in \% aller berufstätigen Ärzte / } \\
\text { en \% de tous les médecins en exercice }\end{array}$ & $13.6 \%$ & $13.4 \%$ & & $40.3 \%$ & $40.8 \%$ & & $53.9 \%$ & $54.2 \%$ & \\
\hline Ohne Praxistätigkeit / Sans pratique privée & $5^{\prime} 756$ & $5^{\prime} 515$ & $+4.4 \%$ & $7 ' 524$ & $7^{\prime} 423$ & $+1.4 \%$ & $13^{\prime} 280$ & $12^{\prime} 938$ & $+2.6 \%$ \\
\hline in $\% /$ en $\%$ & $43.3 \%$ & $42.6 \%$ & & $56.7 \%$ & $57.4 \%$ & & & & \\
\hline $\begin{array}{r}\text { in \% aller berufstätigen Ärzte / } \\
\text { en \% de tous les médecins en exercice }\end{array}$ & $20.0 \%$ & $19.5 \%$ & & $26.1 \%$ & $26.3 \%$ & & $46.1 \%$ & $45.8 \%$ & \\
\hline Total Berufstätige / en exercice & 9'682 & 9'291 & $+4.2 \%$ & $19^{\prime} 130$ & $18 ' 960$ & $+0.9 \%$ & $28 ' 812$ & $28^{\prime} 251$ & $+2.0 \%$ \\
\hline in $\% /$ en $\%$ & $33.6 \%$ & $32.9 \%$ & & $66.4 \%$ & $67.1 \%$ & & & & \\
\hline
\end{tabular}

(c) FMH-Generalsekretariat 2007, Stand per 31.12.2006 


\section{Definition der Begriffe}

Die Statistik enthält Angaben über die berufstätigen Ärztinnen und Ärzte in der Schweiz. Es sind sowohl FMH-Mitglieder als auch Nichtmitglieder enthalten.

\section{Anzahl Einwohner}

Mittlere Wohnbevölkerung gemäss Bundesamt für Statistik, Sektion Bevölkerungsentwicklung.

\section{Ärzte in der Schweiz}

Ärzte, die ihre berufliche Tätigkeit in der Schweiz ausüben.

\section{Ärzte mit Praxistätigkeit}

Ärzte, die in irgendeiner Form eine Praxistätigkeit ausüben, d.h. Ärzte, die in eigener medizinischer Verantwortung Patienten behandeln, sowie Ärzte mit Spitaltätigkeit, die ihr Einkommen ganz oder teilweise aus Patientenhonoraren beziehen, beispielsweise also:

- Ärzte mit privater Praxistätigkeit (Voll- und Teilzeit);

- Spitalärzte (Chef-, Leitende, Beleg-, gegebenenfalls Oberärzte) mit Praxistätigkeit;

- Ärzte mit Praxistätigkeit, die zusätzlich in irgendeiner Form auch angestellt sind;

- angestellte Ärzte, die zusätzlich eine Praxistätigkeit ausüben.

\section{Ärzte ohne Praxistätigkeit}

Berufstätige Ärzte, die nicht in der Kategorie «Ärzte mit Praxistätigkeit» enthalten sind:

- Chefärzte;

- Leitende Ärzte;

- Oberärzte;

- Assistenten;

- andere Tätigkeit (Verwaltung, Industrie, Versicherungen usw.).

\section{Ärzte ohne Facharzttitel}

Ärzte, die keinen Facharzttitel führen.

\section{Belegärzte}

Ärzte mit Praxistätigkeit, die in irgendeiner vertraglich vereinbarten Form die Spitalinfrastruktur in Anspruch nehmen und/oder vertraglich vereinbarte Dienstleistungen gegenüber dem Spital erbringen.

\section{Chefärzte}

Ärzte, die am Spital als Chefärzte angestellt sind (mit oder ohne Praxistätigkeit).

\section{Durchschnittsalter}

Summe des Alters dividiert durch Anzahl Ärzte.

\section{Fachärzte}

Ärzte, die einen oder mehrere Facharzttitel führen.

\section{Kanton}

Als Kanton gilt der Standortkanton der Praxis oder der beruflichen Haupttätigkeit. Wenn keine der beiden Angaben bekannt ist, gilt der Standortkanton der Kontaktadresse.

\section{Leitende Ärzte}

Ärzte, die am Spital in leitender Position angestellt sind (mit oder ohne Praxistätigkeit).

\section{MS-Region}

Regionale Gliederung der Schweiz gemäss Programm NFP-5 des Schweizerischen Nationalfonds. MS bedeutet «mobilité spatiale».

\section{Sterbealter}

Summe des Alters zum Zeitpunkt des Ablebens dividiert durch Anzahl der verstorbenen Ärzte. 


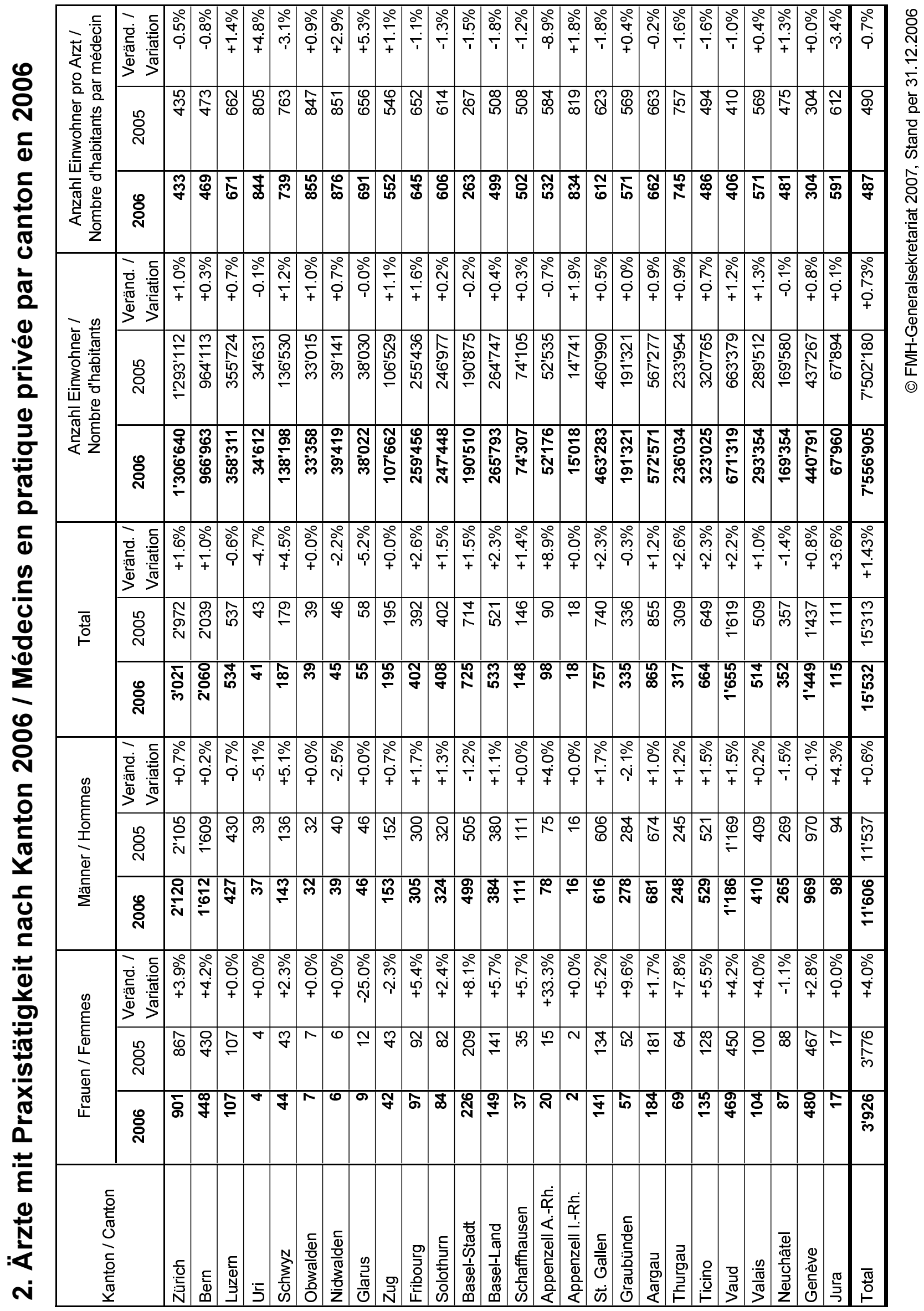




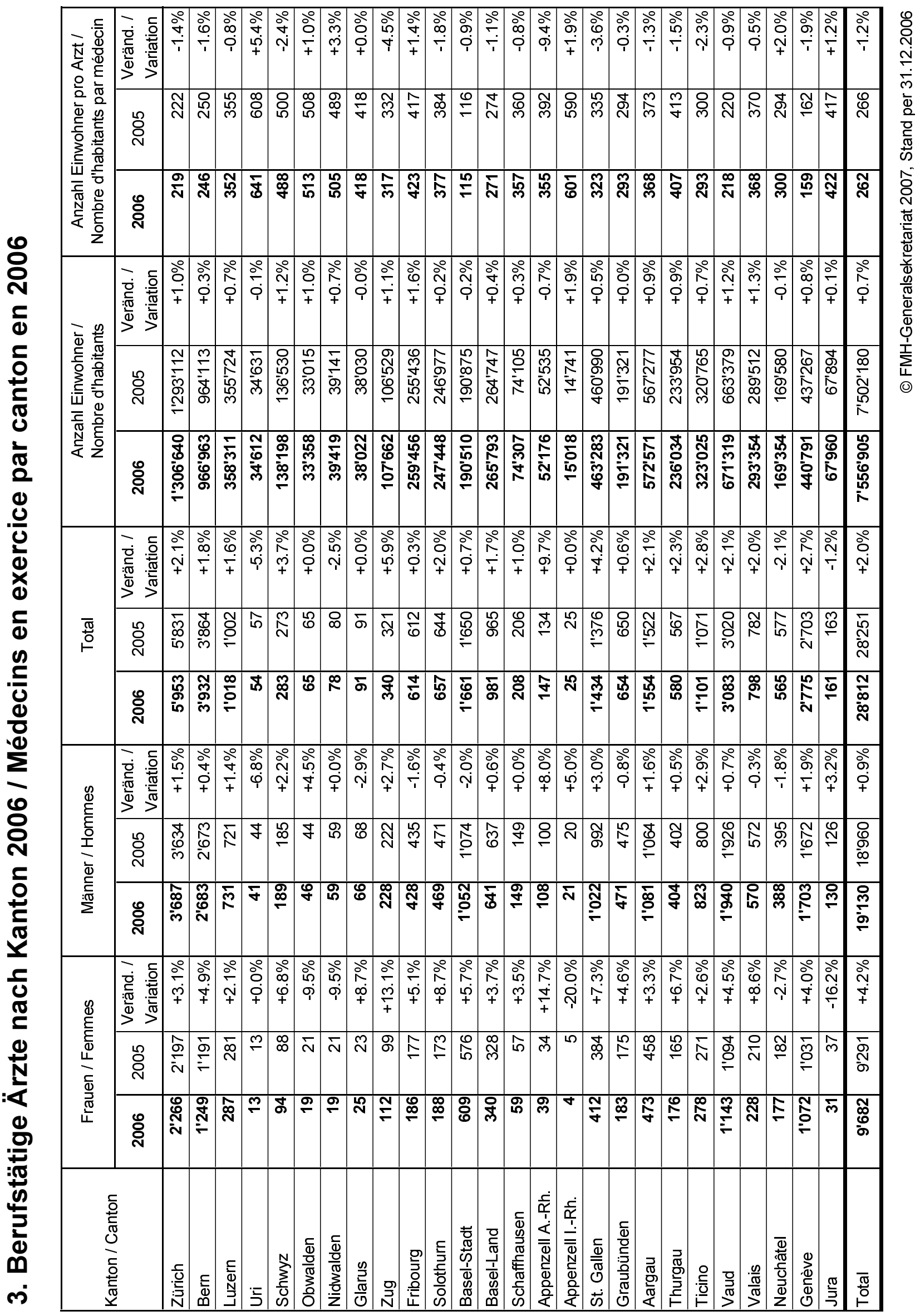




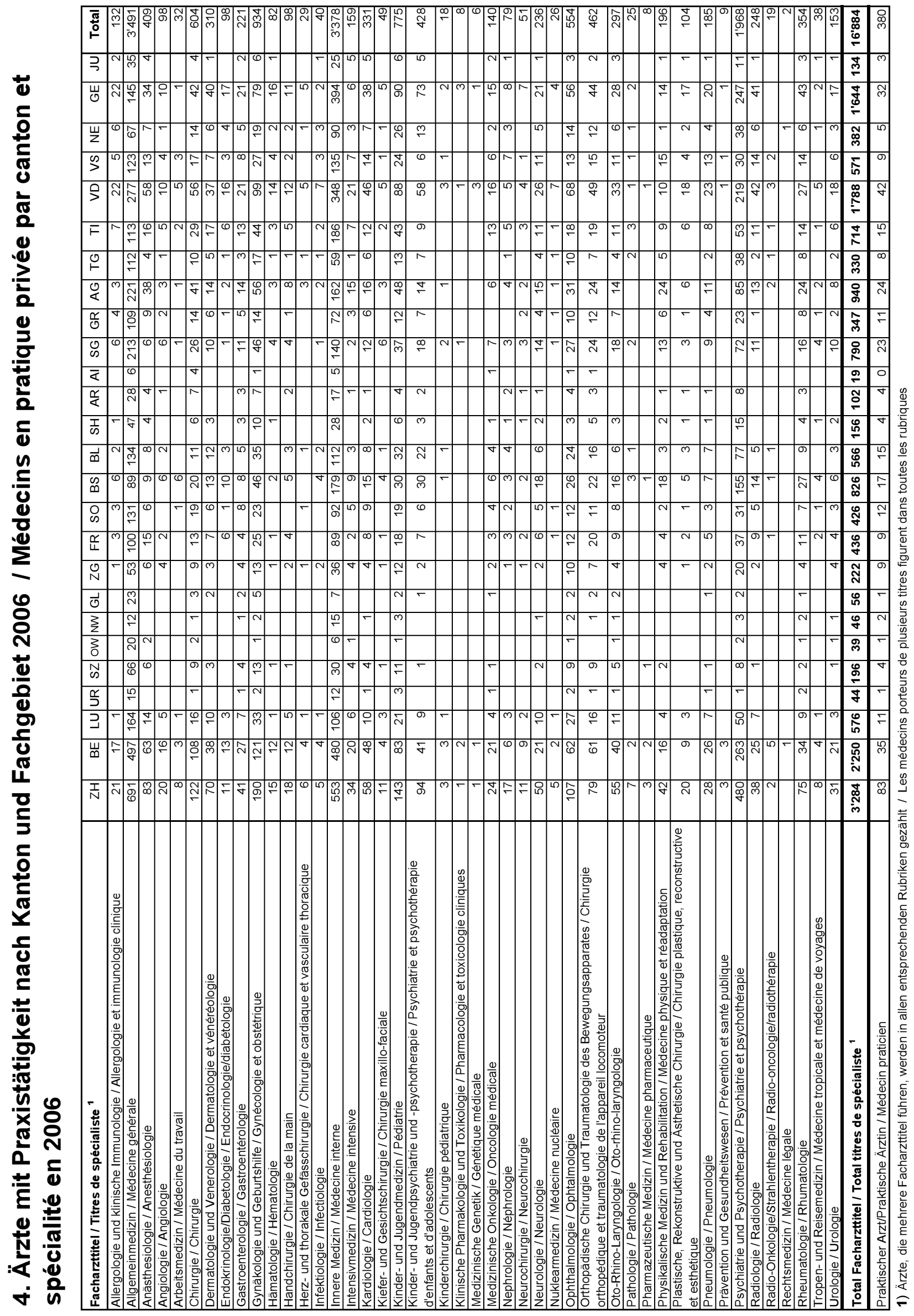




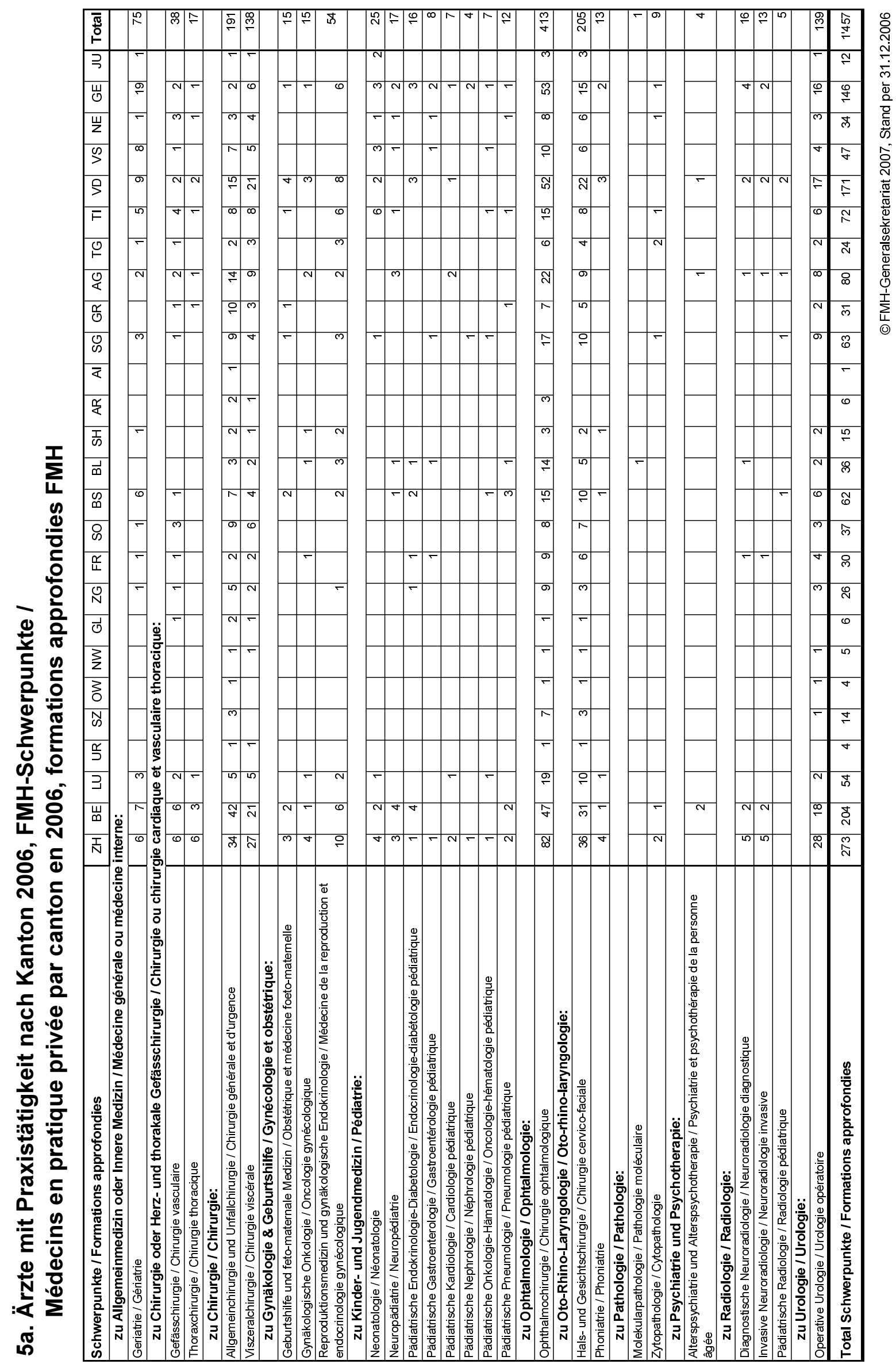




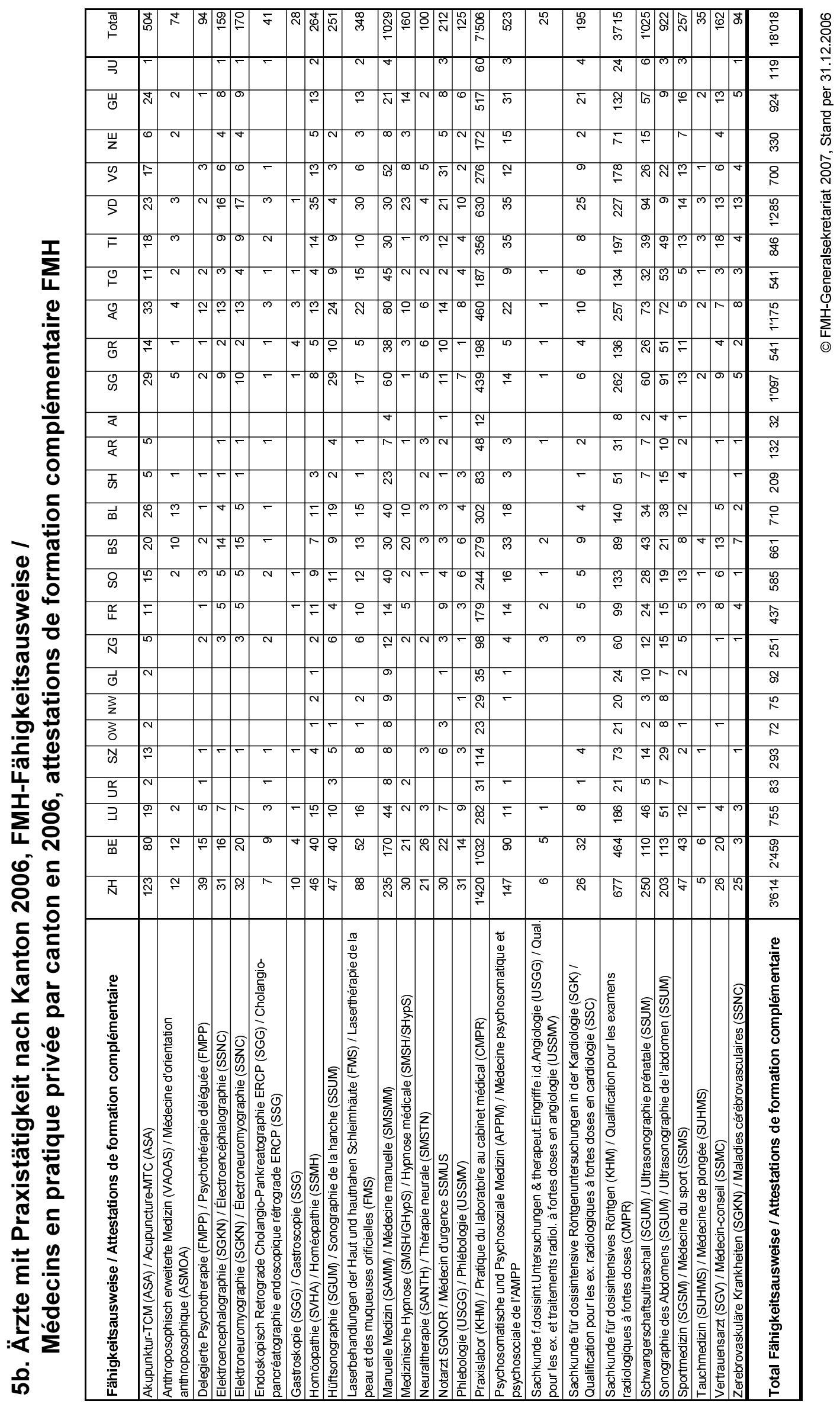




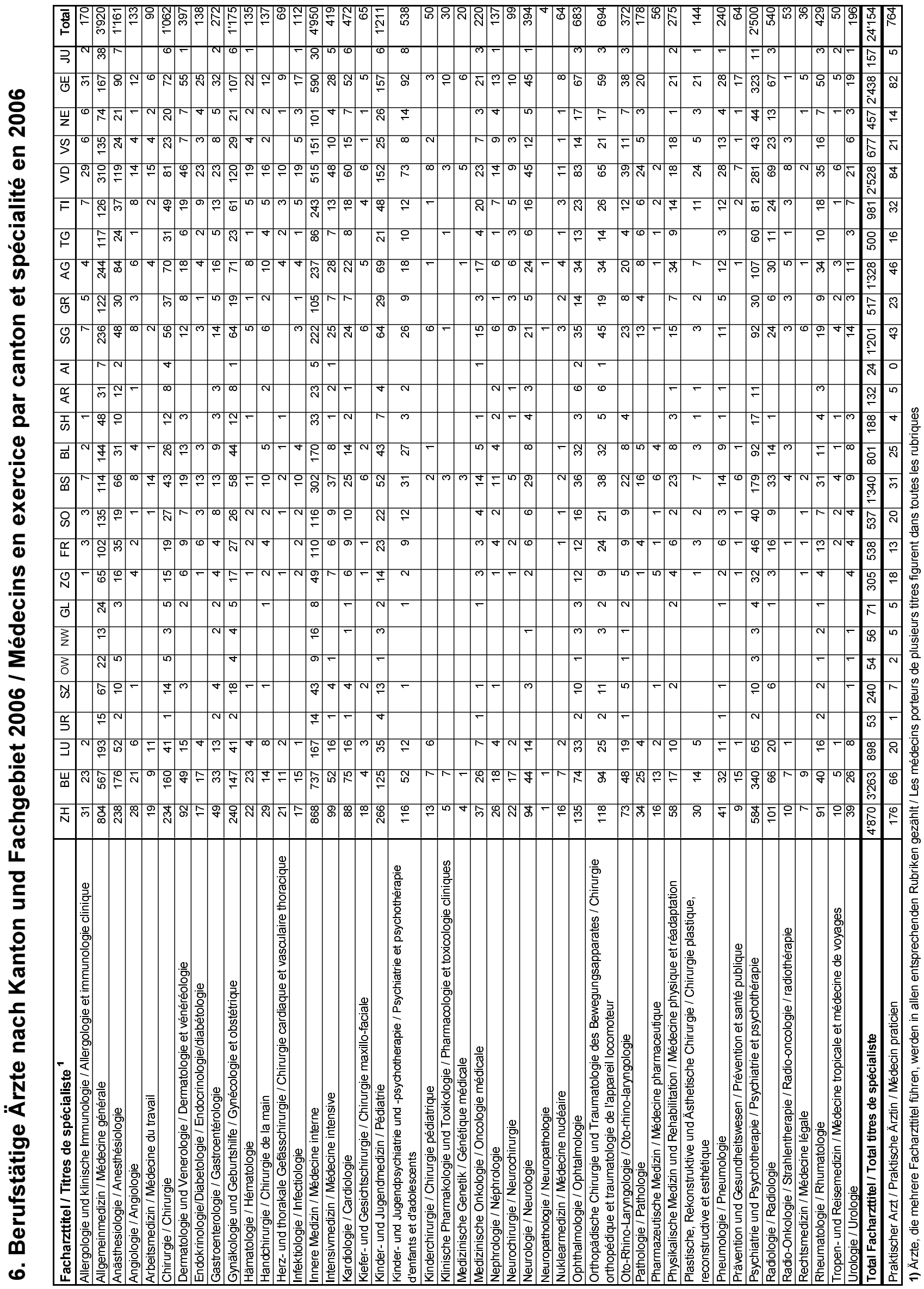




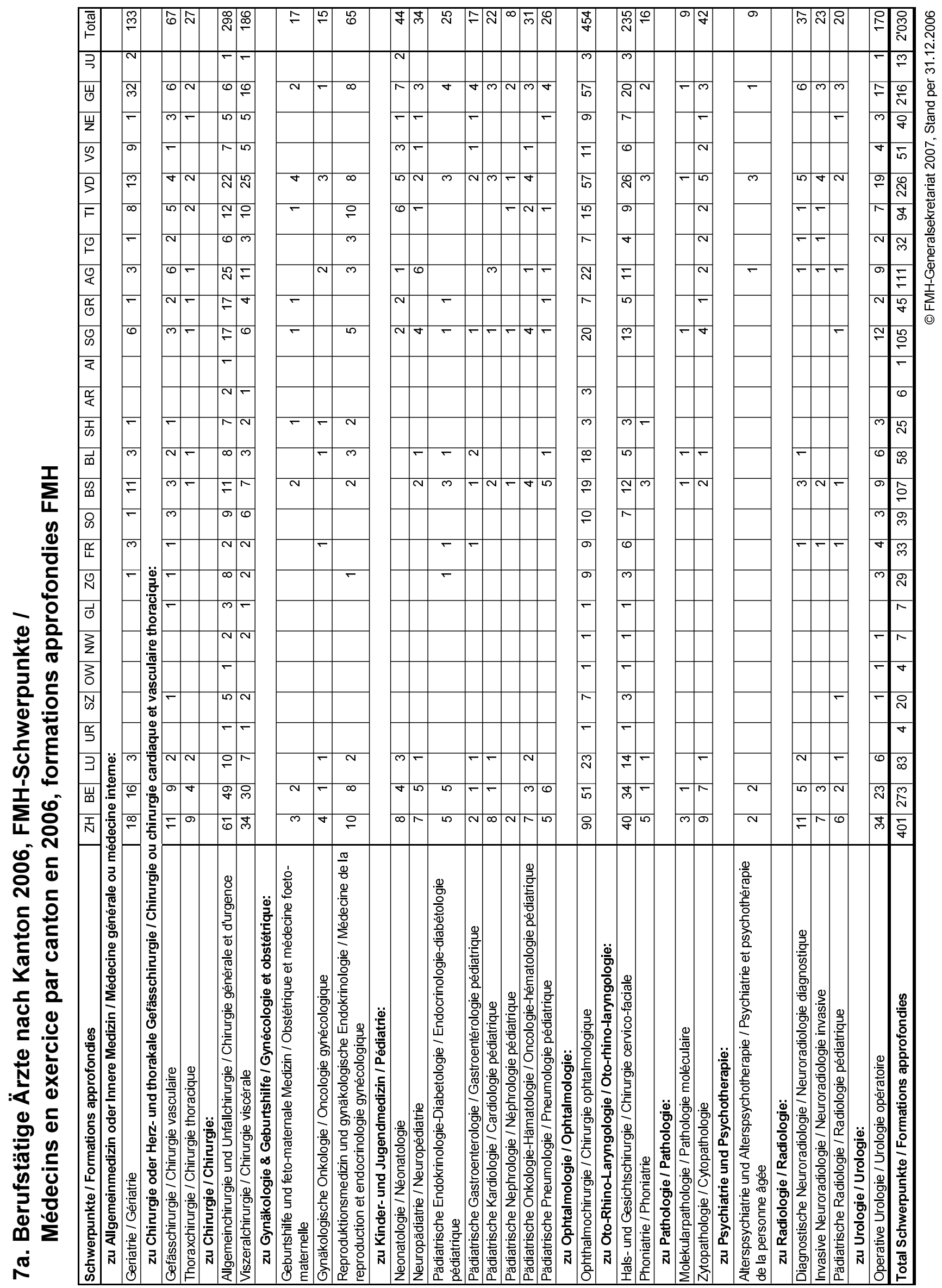




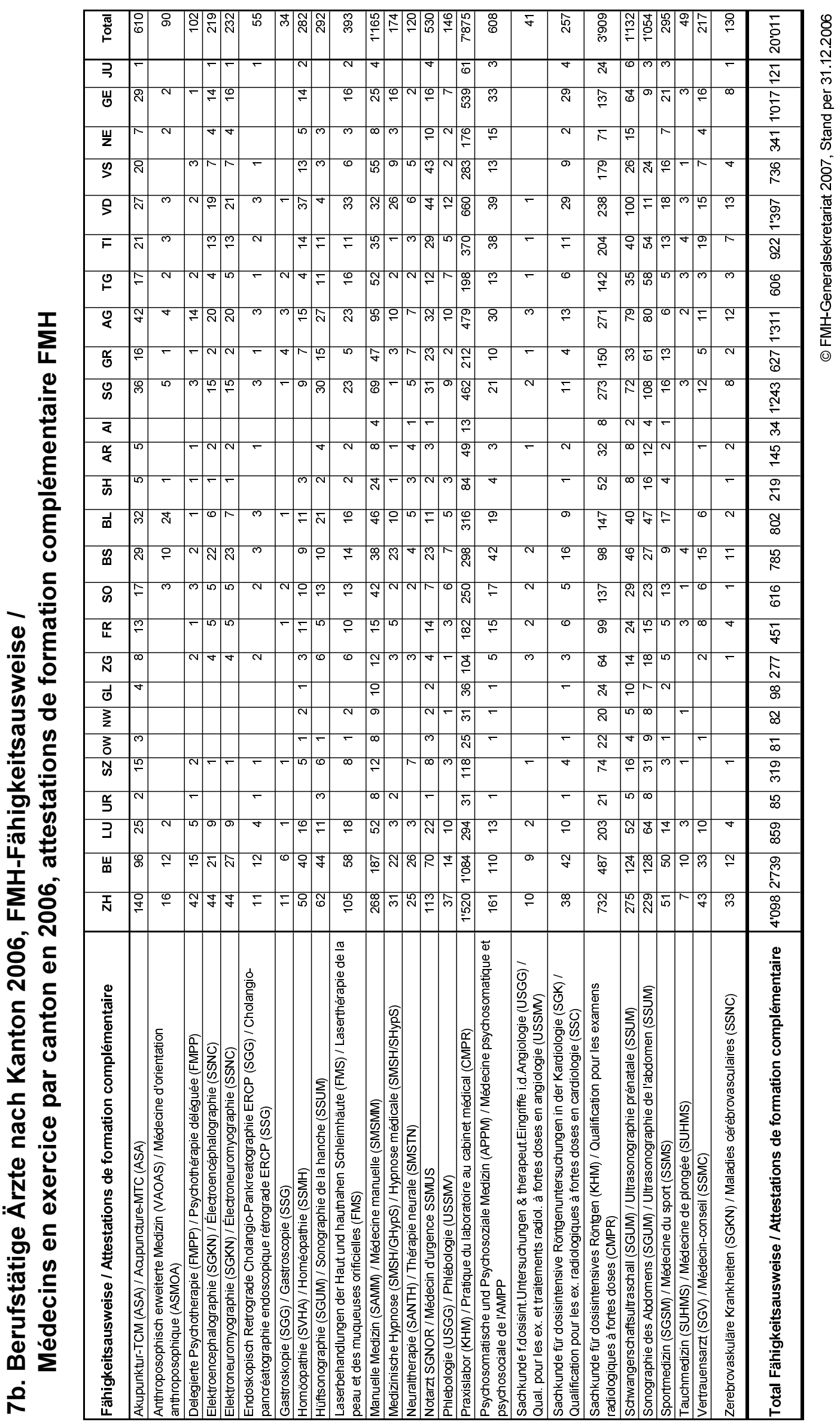




\section{8. Ärzte mit Praxistätigkeit nach Fachgebiet 2006 I Médecins en pratique privée par spécialité en 2006}

\begin{tabular}{|c|c|c|c|c|c|}
\hline Facharzttitel / Titres de spécialiste ${ }^{1}$ & $\begin{array}{l}\text { Frauen / } \\
\text { Femmes }\end{array}$ & $\begin{array}{l}\text { in } \% / \\
\text { en } \%\end{array}$ & $\begin{array}{l}\text { Männer / } \\
\text { Hommes }\end{array}$ & $\begin{array}{l}\text { in } \% / \\
\text { en } \%\end{array}$ & Total \\
\hline Allergologie und klinische Immunologie / Allergologie et immunologie clinique & 32 & $24 \%$ & 100 & $76 \%$ & 132 \\
\hline Allgemeinmedizin / Médecine générale & 642 & $18 \%$ & 2849 & $82 \%$ & 3'491 \\
\hline Anästhesiologie / Anesthésiologie & 110 & $27 \%$ & 299 & $73 \%$ & 409 \\
\hline Angiologie / Angiologie & 15 & $15 \%$ & 83 & $85 \%$ & 98 \\
\hline Arbeitsmedizin / Médecine du travail & 7 & $22 \%$ & 25 & $78 \%$ & 32 \\
\hline Chirurgie / Chirurgie & 27 & $4 \%$ & 577 & $96 \%$ & 604 \\
\hline Dermatologie und Venerologie / Dermatologie et vénéréologie & 122 & $39 \%$ & 188 & $61 \%$ & 310 \\
\hline Endokrinologie/Diabetologie / Endocrinologie/diabétologie & 26 & $27 \%$ & 72 & $73 \%$ & 98 \\
\hline Gastroenterologie / Gastroentérologie & 9 & $4 \%$ & 212 & $96 \%$ & 221 \\
\hline Gynäkologie und Geburtshilfe / Gynécologie et obstétrique & 320 & $34 \%$ & 614 & $66 \%$ & 934 \\
\hline Hämatologie / Hématologie & 18 & $22 \%$ & 64 & $78 \%$ & 82 \\
\hline Handchirurgie / Chirurgie de la main & 16 & $16 \%$ & 82 & $84 \%$ & 98 \\
\hline Herz- und thorakale Gefässchirurgie / Chirurgie cardiaque et vasculaire thoracique & 1 & $3 \%$ & 28 & $97 \%$ & 29 \\
\hline Infektiologie / Infectiologie & 3 & $8 \%$ & 37 & $93 \%$ & 40 \\
\hline Innere Medizin / Médecine interne & 607 & $18 \%$ & 2771 & $82 \%$ & 3'378 \\
\hline Intensivmedizin / Médecine intensive & 19 & $12 \%$ & 140 & $88 \%$ & 159 \\
\hline Kardiologie / Cardiologie & 30 & $9 \%$ & 301 & $91 \%$ & 331 \\
\hline Kiefer- und Gesichtschirurgie / Chirurgie maxillo-faciale & 3 & $6 \%$ & 46 & $94 \%$ & 49 \\
\hline Kinder- und Jugendmedizin / Pédiatrie & 307 & $40 \%$ & 468 & $60 \%$ & 775 \\
\hline $\begin{array}{l}\text { Kinder- und Jugendpsychiatrie und -psychotherapie / Psychiatrie et psychothérapie } \\
\text { d'enfants et d'adolescents }\end{array}$ & 242 & $57 \%$ & 186 & $43 \%$ & 428 \\
\hline Kinderchirurgie / Chirurgie pédiatrique & 5 & $28 \%$ & 13 & $72 \%$ & 18 \\
\hline Klinische Pharmakologie und Toxikologie / Pharmacologie et toxicologie cliniques & 2 & $25 \%$ & 6 & $75 \%$ & 8 \\
\hline Medizinische Genetik / Génétique médicale & 2 & $33 \%$ & 4 & $67 \%$ & 6 \\
\hline Medizinische Onkologie / Oncologie médicale & 33 & $24 \%$ & 107 & $76 \%$ & 140 \\
\hline Nephrologie / Néphrologie & 12 & $15 \%$ & 67 & $85 \%$ & 79 \\
\hline Neurochirurgie / Neurochirurgie & 1 & $2 \%$ & 50 & $98 \%$ & 51 \\
\hline Neurologie / Neurologie & 46 & $19 \%$ & 190 & $81 \%$ & 236 \\
\hline Nuklearmedizin / Médecine nucléaire & 5 & $19 \%$ & 21 & $81 \%$ & 26 \\
\hline Ophthalmologie / Ophtalmologie & 198 & $36 \%$ & 356 & $64 \%$ & 554 \\
\hline $\begin{array}{l}\text { Orthopädische Chirurgie und Traumatologie des Bewegungsapparates / Chirurgie } \\
\text { orthopédique et traumatologie de l'appareil locomoteur }\end{array}$ & 20 & $4 \%$ & 442 & $96 \%$ & 462 \\
\hline Oto-Rhino-Laryngologie / Oto-rhino-laryngologie & 47 & $16 \%$ & 250 & $84 \%$ & 297 \\
\hline Pathologie / Pathologie & 6 & $24 \%$ & 19 & $76 \%$ & 25 \\
\hline Pharmazeutische Medizin / Médecine pharmaceutique & 1 & $13 \%$ & 7 & $88 \%$ & 8 \\
\hline Physikalische Medizin und Rehabilitation / Médecine physique et réadaptation & 34 & $17 \%$ & 162 & $83 \%$ & 196 \\
\hline $\begin{array}{l}\text { Plastische, Rekonstruktive und Ästhetische Chirurgie / Chirurgie plastique, } \\
\text { reconstructive et esthétique }\end{array}$ & 24 & $23 \%$ & 80 & $77 \%$ & 104 \\
\hline Pneumologie / Pneumologie & 14 & $8 \%$ & 171 & $92 \%$ & 185 \\
\hline Prävention und Gesundheitswesen / Prévention et santé publique & 0 & $0 \%$ & 9 & $100 \%$ & 9 \\
\hline Psychiatrie und Psychotherapie / Psychiatrie et psychothérapie & 742 & $38 \%$ & 1226 & $62 \%$ & $1 ' 968$ \\
\hline Radiologie / Radiologie & 47 & $19 \%$ & 201 & $81 \%$ & 248 \\
\hline Radio-Onkologie / Strahlentherapie / Radio-oncologie / radiothérapie & 8 & $42 \%$ & 11 & $58 \%$ & 19 \\
\hline Rechtsmedizin / Médecine légale & 0 & $0 \%$ & 2 & $100 \%$ & 2 \\
\hline Rheumatologie / Rhumatologie & 69 & $19 \%$ & 285 & $81 \%$ & 354 \\
\hline Tropen- und Reisemedizin / Médecine tropicale et médecine de voyages & 5 & $13 \%$ & 33 & $87 \%$ & 38 \\
\hline Urologie / Urologie & 3 & $2 \%$ & 150 & $98 \%$ & 153 \\
\hline Total Facharzttitel / Total titres de spécialiste ${ }^{1}$ & $3^{\prime} 880$ & $23 \%$ & $13^{\prime} 004$ & $77 \%$ & $16 ' 884$ \\
\hline Praktischer Arzt/Praktische Ärztin / Médecin praticien & 197 & $52 \%$ & 183 & $48 \%$ & 380 \\
\hline
\end{tabular}

1) Ärzte, die mehrere Facharzttitel führen, werden in allen entsprechenden Rubriken gezählt / Les médecins porteurs de plusieurs titres figurent dans toutes les rubriques 


\section{9. Ärzte mit Praxistätigkeit 2006, FMH-Schwerpunkte und FMH-Ausweise I Médecins en pratique privée en 2006, formations approfondies et formation complémentaire FMH}

\begin{tabular}{|c|c|c|c|c|c|}
\hline & \begin{tabular}{|c|} 
Frauen $/$ \\
Femmes \\
\end{tabular} & \begin{tabular}{rl|} 
in $\% /$ \\
en $\%$ \\
\end{tabular} & $\begin{array}{r}\text { Männer I } \\
\text { Hommes }\end{array}$ & $\begin{array}{r}\text { in } \% ~ \\
\text { en } \% \\
\end{array}$ & Total \\
\hline \multicolumn{6}{|l|}{ Schwerpunkte / Formations approfondies: } \\
\hline \multicolumn{6}{|l|}{ zu Allgemeinmedizin oder Innere Medizin / Médecine générale ou médecine interme: } \\
\hline Geriatrie / gériatrie & 10 & $13 \%$ & 65 & $87 \%$ & 7 \\
\hline \multicolumn{6}{|c|}{ zu Chirurgie oder Herz- und thorakale Gefässchirurgie / Chirurgie ou chirurgie cardiaque et vasculaire thoracique: } \\
\hline Gefässchirurgie / Chirurgie vasculaire & 1 & $3 \%$ & 37 & $97 \%$ & \\
\hline Thoraxchirurgie / Chirurgie thoracique & 0 & $0 \%$ & 17 & $100 \%$ & 17 \\
\hline \multicolumn{6}{|l|}{ zu Chirurgie / Chirurgie: } \\
\hline Allgemeinchirurgie und Unfallchirurgie / Chirurgie générale et d'urgence & 5 & $3 \%$ & 186 & $97 \%$ & 191 \\
\hline Viszeralchirurgie / Chirurgie viscérale & 2 & $1 \%$ & 136 & $99 \%$ & 138 \\
\hline \multicolumn{6}{|l|}{ zu Gynäkologie \& Geburtshilfe / Gynécologie et obstétrique: } \\
\hline Geburtshilfe und feto-matemale Medizin / Obstétrique et médecine foeto-matemelle & 2 & $13 \%$ & 13 & $87 \%$ & \\
\hline Gynäkologische Onkologie / Oncologie gynécologique & 0 & $0 \%$ & 15 & $100 \%$ & 15 \\
\hline $\begin{array}{l}\text { Reproduktionsmedizin und gynäkologische Endokrinologie / Médecine de la reproduction et endocrinologie } \\
\text { gynécologique }\end{array}$ & 16 & $30 \%$ & 38 & $70 \%$ & 5 \\
\hline \multicolumn{6}{|l|}{ zu Kinder- und Jugendmedizin / Pédiatrie: } \\
\hline Neoantologie / Néonatologie & 4 & $16 \%$ & 21 & $84 \%$ & 25 \\
\hline Neuropädiatrie / Neuropédiatrie & 7 & $41 \%$ & 10 & $59 \%$ & 17 \\
\hline Pädiatrische Endokrinologie-Diabetologie / Endocrinologie-diabétologie pédiatrique & 4 & $25 \%$ & 12 & $75 \%$ & 16 \\
\hline Pädiatrische Gastroenterologie / Gastroentérologie pédiatrique & 0 & $0 \%$ & 8 & $100 \%$ & \\
\hline Pädiatrische Kardiologie / Cardiologie pédiatrique & 0 & $0 \%$ & 7 & $100 \%$ & \\
\hline Pädiatrische Nephrologie / Néphrologie pédiatrique & 2 & $50 \%$ & 2 & $50 \%$ & \\
\hline Pädiatrische Onkologie-Hämatologie / Oncologie-hématologie pédiatrique & 3 & $43 \%$ & 4 & $57 \%$ & \\
\hline Pädiatrische Pneumologie / Pneumologie pédiatrique & 4 & $33 \%$ & 8 & $67 \%$ & 12 \\
\hline \multicolumn{6}{|l|}{ zu Ophtalmologie / Ophtalmologie: } \\
\hline Ophthalmochirurgie / Ophtalmochirurgie & 133 & $32 \%$ & 280 & $68 \%$ & 413 \\
\hline \multicolumn{6}{|l|}{ zu Oto-Rhino-Laryngologie / Oto-rhino-laryngologie: } \\
\hline Hals- und Gesichtschirurgie / Chirurgie cervico-faciale & 20 & $10 \%$ & 185 & $90 \%$ & 205 \\
\hline Phoniatrie / Phoniatrie & 7 & $54 \%$ & 6 & $46 \%$ & 13 \\
\hline \multicolumn{6}{|l|}{ zu Pathologie / Pathologie: } \\
\hline Molekularpathologie / Pathologie moléculaire & 0 & $0 \%$ & 1 & $100 \%$ & \\
\hline Zytopathologie / Cytopathologie & 3 & $33 \%$ & 6 & $67 \%$ & \\
\hline \multicolumn{6}{|l|}{ zu Psychiatrie und Psychotherapie: } \\
\hline Alterspsychiatrie und Alterspsychotherapie / Psychiatrie et psychothérapie de la personne âgée & 1 & $25 \%$ & $3 \mid$ & $75 \%$ & \\
\hline \multicolumn{6}{|l|}{ zu Radiologie / Radiologie: } \\
\hline Diagnostische Neuroradiologie / Neuroradiologie diagnostique & 2 & $13 \%$ & 14 & $88 \%$ & 16 \\
\hline Invasive Neuroradiologie / Neuroradiologie invasive & 2 & $15 \%$ & 11 & $85 \%$ & 13 \\
\hline Pädiatrische Radiologie / Radiologie pédiatrique & 1 & $20 \%$ & 4 & $80 \%$ & \\
\hline \multicolumn{6}{|l|}{ zu Urologie / Urologie: } \\
\hline Operative Urologie / Urologie opératoire & 2 & $1 \%$ & 137 & $99 \%$ & 139 \\
\hline Total Schwerpunkte / Formations approfondies & 231 & $16 \%$ & $1 ' 226$ & $84 \%$ & 14457 \\
\hline
\end{tabular}

Fähigkeitsausweise / Attestations de formation complémentaire:

Akupunktur-TCM (ASA) / Acupuncture-MTC (ASA)

Anthroposophisch erweiterte Medizin (VAOAS) / Médecine d'orientation anthroposophique (ASMOA)

Delegierte Psychotherapie (FMPP) / Psychothérapie déléguée (FMPP)

Elektroencephalographie (SGKN) / Électroencephalographie (SSNC)

Elektroneuromyographie (SGKN) / Électroneuromyographie (SSNC)

Endoskopisch Retrograde Cholangio-Pankreatographie ERCP (SGG) / Cholangio-pancréatographie

endoscopique rétrograde ERCP (SSG)

Gastroskopie (SGG) / Gastroscopie (SSG)

Homöopathie (SVHA) / Homéopathie (SSMH)

Hüftsonographie (SGUM) / Sonographie de la hanche (SSUM)

Laserbehandlungen der Haut und hautnahen Schleimhäute (FMS) / Laserthérapie de la peau et des

muqueuses orificielles (FMS)

Manuelle Medizin (SAMM) / Médecine manuelle (SMSMM)

Medizinische Hypnose (SMSH/GHypS) / Hypnose médicale (SMSH/SHypS)

Neuraltherapie (SANTH) / Thérapie neurale (SMSTN)

Notarzt SGNOR / Médecin d'urgence SSMUS

Phlebologie (USGG) / Phlébologie (USSMV)

Praxislabor (KHM) / Pratique du laboratoire au cabinet médical (CMPR)

Psychosomatische und Psychosoziale Medizin (APPM) / Médecine psychosomatique et psychosociale de

I'AMPP

Sachkunde f.dosisint.Untersuchungen \& therapeut.Eingriffe i.d.Angiologie (USGG) / Qual. pour les ex. et traitements radiol. à fortes doses en angiologie (USSMV)

Sachkunde für dosisintensive Röntgenuntersuchungen in der Kardiologie (SGK) / Qualification pour les ex. radiologiques à fortes doses en cardiologie (SSC)

Sachkunde für dosisintensives Röntgen (KHM) / Qualification pour les examens radiologiques à fortes doses (CMPR)

Schwangerschaftsultraschall (SGUM) / Ultrasonographie prénatale (SSUM)

Sonographie des Abdomens (SGUM) / Ultrasonographie de l'abdomen (SSUM)

Sportmedizin (SGSM) / Médecine du sport (SSMS)

Tauchmedizin (SUHMS) / Médecine de plongée (SUHMS

Vertrauensarzt (SGV) / Médecin-conseil (SSMC)

Zerebrovaskuläre Krankheiten (SGKN) / Maladies cérébrovasculaires (SSNC)

Total Fähigkeitsausweise / Attestations de formation complémentaire

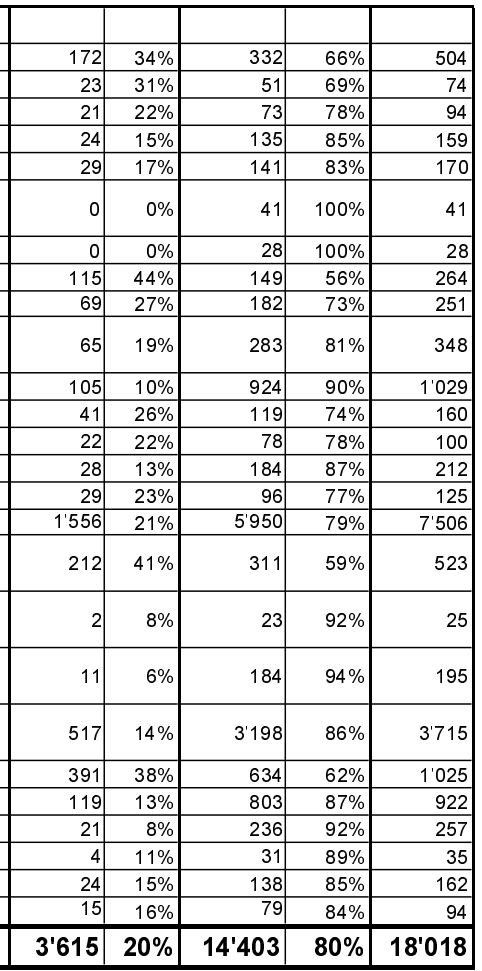

(c) FMH-Generalsekretariat 2007, Stand per 31.12.2006 


\section{Berufstätige Ärzte nach Fachgebiet 2006 / Médecins en exercice par spécialité en 2006}

\begin{tabular}{|c|c|c|c|c|c|}
\hline Facharzttitel / Titres de spécialiste ${ }^{1}$ & $\begin{array}{l}\text { Frauen } 1 \\
\text { Femmes }\end{array}$ & $\begin{array}{l}\text { in \% } \\
\text { en } \%\end{array}$ & $\begin{array}{l}\text { Männer } / \\
\text { Hommes }\end{array}$ & \begin{tabular}{|c|} 
in \% \\
en \%
\end{tabular} & Total \\
\hline Allergologie und klinische Immunologie / Allergologie et immunologie clinique & 43 & $25 \%$ & 127 & $75 \%$ & 170 \\
\hline Allgemeinmedizin / Médecine générale & 809 & $21 \%$ & $3^{\prime} 111$ & $79 \%$ & 3 '920 \\
\hline Anästhesiologie / Anesthésiologie & 424 & $37 \%$ & 737 & $63 \%$ & 1'161 \\
\hline Angiologie / Angiologie & 26 & $20 \%$ & 107 & $80 \%$ & 133 \\
\hline Arbeitsmedizin / Médecine du travail & 24 & $27 \%$ & 66 & $73 \%$ & 90 \\
\hline Chirurgie / Chirurgie & 90 & $8 \%$ & 972 & $92 \%$ & 1 '062 \\
\hline Dermatologie und Venerologie / Dermatologie et vénéréologie & 154 & $39 \%$ & 243 & $61 \%$ & 397 \\
\hline Endokrinologie/Diabetologie / Endocrinologie/diabétologie & 34 & $25 \%$ & 104 & $75 \%$ & 138 \\
\hline Gastroenterologie / Gastroentérologie & 19 & $7 \%$ & 253 & $93 \%$ & 272 \\
\hline Gynäkologie und Geburtshilfe / Gynécologie et obstétrique & 474 & $40 \%$ & 701 & $60 \%$ & 1'175 \\
\hline Hämatologie / Hématologie & 36 & $27 \%$ & 99 & $73 \%$ & 135 \\
\hline Handchirurgie / Chirurgie de la main & 24 & $18 \%$ & 113 & $82 \%$ & 137 \\
\hline $\begin{array}{l}\text { Herz- und thorakale Gefässchirurgie / Chirurgie cardiaque et vasculaire } \\
\text { thoracique }\end{array}$ & 3 & $4 \%$ & 66 & $96 \%$ & 69 \\
\hline Infektiologie / Infectiologie & 18 & $16 \%$ & 94 & $84 \%$ & 112 \\
\hline Innere Medizin / Médecine interne & $1 ' 170$ & $24 \%$ & 3780 & $76 \%$ & 4 4'950 \\
\hline Intensivmedizin / Médecine intensive & 99 & $24 \%$ & 320 & $76 \%$ & 419 \\
\hline Kardiologie / Cardiologie & 51 & $11 \%$ & 421 & $89 \%$ & 472 \\
\hline Kiefer- und Gesichtschirurgie / Chirurgie maxillo-faciale & 5 & $8 \%$ & 60 & $92 \%$ & 65 \\
\hline Kinder- und Jugendmedizin / Pédiatrie & 547 & $45 \%$ & 664 & $55 \%$ & 1 1'211 \\
\hline $\begin{array}{l}\text { Kinder- und Jugendpsychiatrie und -psychotherapie / Psychiatrie et } \\
\text { psychothérapie d'enfants et d'adolescents }\end{array}$ & 306 & $57 \%$ & 232 & $43 \%$ & 538 \\
\hline Kinderchirurgie / Chirurgie pédiatrique & 13 & $26 \%$ & 37 & $74 \%$ & 50 \\
\hline $\begin{array}{l}\text { Klinische Pharmakologie und Toxikologie / Pharmacologie et toxicologie } \\
\text { cliniques }\end{array}$ & 9 & $30 \%$ & 21 & $70 \%$ & 30 \\
\hline Medizinische Genetik / Génétique médicale & 8 & $40 \%$ & 12 & $60 \%$ & 20 \\
\hline Medizinische Onkologie / Oncologie médicale & 56 & $25 \%$ & 164 & $75 \%$ & 220 \\
\hline Nephrologie / Néphrologie & 34 & $25 \%$ & 103 & $75 \%$ & 137 \\
\hline Neurochirurgie / Neurochirurgie & 11 & $11 \%$ & 88 & $89 \%$ & 99 \\
\hline Neurologie / Neurologie & 96 & $24 \%$ & 298 & $76 \%$ & 394 \\
\hline Neuropathologie / Neuropathologie & 0 & $0 \%$ & 4 & $100 \%$ & 4 \\
\hline Nuklearmedizin / Médecine nucléaire & 11 & $17 \%$ & 53 & $83 \%$ & 64 \\
\hline Ophthalmologie / Ophtalmologie & 253 & $37 \%$ & 430 & $63 \%$ & 683 \\
\hline $\begin{array}{l}\text { Orthopädische Chirurgie und Traumatologie des Bewegungsapparates / } \\
\text { Chirurgie orthopédique et traumatologie de l'appareil locomoteur }\end{array}$ & 35 & $5 \%$ & 659 & $95 \%$ & 694 \\
\hline Oto-Rhino-Laryngologie / Oto-rhino-laryngologie & 72 & $19 \%$ & 300 & $81 \%$ & 372 \\
\hline Pathologie / Pathologie & 75 & $42 \%$ & 103 & $58 \%$ & 178 \\
\hline Pharmazeutische Medizin / Médecine pharmaceutique & 19 & $34 \%$ & 37 & $66 \%$ & 56 \\
\hline Physikalische Medizin und Rehabilitation / Médecine physique et réadaptation & 63 & $23 \%$ & 212 & $77 \%$ & 275 \\
\hline $\begin{array}{l}\text { Plastische, Rekonstruktive und Ästhetische Chirurgie / Chirurgie plastique, } \\
\text { reconstructive et esthétique }\end{array}$ & 36 & $25 \%$ & 108 & $75 \%$ & 144 \\
\hline Pneumologie / Pneumologie & 27 & $11 \%$ & 213 & $89 \%$ & 240 \\
\hline Prävention und Gesundheitswesen / Prévention et santé publique & 23 & $36 \%$ & 41 & $64 \%$ & 64 \\
\hline Psychiatrie und Psychotherapie / Psychiatrie et psychothérapie & 956 & $38 \%$ & $11^{\prime} 544$ & $62 \%$ & $2{ }^{\prime} 500$ \\
\hline Radiologie / Radiologie & 126 & $23 \%$ & 414 & $77 \%$ & 540 \\
\hline Radio-Onkologie / Strahlentherapie / Radio-oncologie / radiothérapie & 19 & $36 \%$ & 34 & $64 \%$ & 53 \\
\hline Rechtsmedizin / Médecine légale & 9 & $25 \%$ & 27 & $75 \%$ & 36 \\
\hline Rheumatologie / Rhumatologie & 91 & $21 \%$ & 338 & $79 \%$ & 429 \\
\hline Tropen- und Reisemedizin / Médecine tropicale et médecine de voyages & 5 & $10 \%$ & 45 & $90 \%$ & 50 \\
\hline Urologie / Urologie & 7 & $4 \%$ & 189 & $96 \%$ & 196 \\
\hline Total Facharzttitel / Total titres de spécialiste ${ }^{1}$ & $6^{\prime} 410$ & $27 \%$ & $17^{\prime} 744$ & $73 \%$ & $24^{\prime} 154$ \\
\hline Praktischer Arzt/Praktische Ärztin / Médecin praticien & 377 & $49 \%$ & 387 & $51 \%$ & 764 \\
\hline
\end{tabular}

1) Ärzte, die mehrere Facharzttitel führen, werden in allen entsprechenden Rubriken gezählt / Les médecins porteurs de plusieurs titres figurent dans toutes les rubriques 


\section{Berufstätige Ärzte 2006, FMH-Schwerpunkte und Ausweise I Médecins en exercice en 2006, formations approfondies et certificats FMH}

\begin{tabular}{|c|c|c|c|c|c|}
\hline & \begin{tabular}{|c|} 
Frauen $/$ \\
Femmes \\
\end{tabular} & $\begin{array}{r}\text { in \% ! } \\
\text { en \% } \\
\end{array}$ & $\begin{array}{l}\text { Männer I } \\
\text { Hommes } \\
\end{array}$ & $\begin{array}{r}\text { in \% ! } \\
\text { en \% } \\
\end{array}$ & Total \\
\hline \multicolumn{6}{|l|}{ Schwerpunkte / Formations approfondies: } \\
\hline \multicolumn{6}{|l|}{ zu Allgemeinmedizin oder Innere Medizin / Médecine générale ou médecine interne: } \\
\hline Geriatrie / gériatrie & 33 & $25 \%$ & 100 & $75 \%$ & 133 \\
\hline \multicolumn{6}{|c|}{ zu Chirurgie oder Herz- und thorakale Gefässchirurgie / Chirurgie ou chirurgie cardiaque et vasculaire thoracique: } \\
\hline Gefässchirurgie / Chirurgie vasculaire & 1 & $1 \%$ & 66 & $99 \%$ & 67 \\
\hline Thoraxchirurgie / Chirurgie thoracique & & $0 \%$ & 27 & $100 \%$ & 27 \\
\hline \multicolumn{6}{|l|}{ zu Chirurgie / Chirurgie: } \\
\hline Allgemeinchirurgie und Unfallchirurgie / Chirurgie générale et d'urgence & 18 & $6 \%$ & 280 & $94 \%$ & 298 \\
\hline Viszeralchirurgie / Chirurgie viscérale & 9 & $5 \%$ & 177 & $95 \%$ & 186 \\
\hline \multicolumn{6}{|l|}{ zu Gynäkologie \& Geburtshilfe / Gynécologie et obstétrique: } \\
\hline Geburtshilfe und feto-maternale Medizin / Obstétrique et médecine foeto-maternelle & 2 & $12 \%$ & 15 & $88 \%$ & \\
\hline Gynäkologische Onkologie / Oncologie gynécologique & & $0 \%$ & 15 & $100 \%$ & 15 \\
\hline $\begin{array}{l}\text { Reproduktionsmedizin und gynäkologische Endokrinologie / Médecine de la reproduction et } \\
\text { endocrinologie gynécologique }\end{array}$ & 22 & $34 \%$ & 43 & $66 \%$ & 65 \\
\hline \multicolumn{6}{|l|}{ zu Kinder- und Jugendmedizin / Pédiatrie: } \\
\hline Neoantologie / Néonatologie & 12 & $27 \%$ & 32 & $73 \%$ & 44 \\
\hline Neuropädiatrie / Neuropédiatrie & 16 & $47 \%$ & 18 & $53 \%$ & 34 \\
\hline Pädiatrische Endokrinologie-Diabetologie / Endocrinologie-diabétologie pédiatrique & 8 & $32 \%$ & 17 & $68 \%$ & 25 \\
\hline Pädiatrische Gastroenterologie / Gastroentérologie pédiatrique & 3 & $18 \%$ & 14 & $82 \%$ & 17 \\
\hline Pädiatrische Kardiologie / Cardiologie pédiatrique & 7 & $32 \%$ & 15 & $68 \%$ & 22 \\
\hline Pädiatrische Nephrologie / Néphrologie pédiatrique & 2 & $25 \%$ & 6 & $75 \%$ & \\
\hline Pädiatrische Onkologie-Hämatologie / Oncologie-hématologie pédiatrique & 9 & $29 \%$ & 22 & $71 \%$ & 31 \\
\hline Pädiatrische Pneumologie / Pneumologie pédiatrique & 8 & $31 \%$ & 18 & $69 \%$ & 26 \\
\hline \multicolumn{6}{|l|}{ zu Ophtalmologie / Ophtalmologie: } \\
\hline Ophthalmochirurgie / Ophtalmochirurgie & 147 & $32 \%$ & 307 & $68 \%$ & 454 \\
\hline \multicolumn{6}{|l|}{ zu Oto-Rhino-Laryngologie / Oto-rhino-laryngologie: } \\
\hline Hals- und Gesichtschirurgie / Chirurgie cervico-faciale & 27 & $11 \%$ & 208 & $89 \%$ & 235 \\
\hline Phoniatrie / Phoniatrie & 9 & $56 \%$ & 7 & $44 \%$ & 16 \\
\hline \multicolumn{6}{|l|}{ zu Pathologie / Pathologie: } \\
\hline Molekularpathologie / Pathologie moléculaire & 2 & $22 \%$ & 7 & $78 \%$ & \\
\hline Zytopathologie / Cytopathologie & 18 & $43 \%$ & 24 & $57 \%$ & 42 \\
\hline \multicolumn{6}{|l|}{ zu Psychiatrie und Psychotherapie: } \\
\hline Alterspsychiatrie und Alterspsychotherapie / Psychiatrie et psychothérapie de la personne âgée & 3 & $33 \%$ & 6 & $67 \%$ & \\
\hline \multicolumn{6}{|l|}{ zu Radiologie / Radiologie: } \\
\hline Diagnostische Neuroradiologie / Neuroradiologie diagnostique & 4 & $11 \%$ & 33 & $89 \%$ & 37 \\
\hline Invasive Neuroradiologie / Neuroradiologie invasive & 3 & $13 \%$ & 20 & $87 \%$ & 23 \\
\hline Pädiatrische Radiologie / Radiologie pédiatrique & 7 & $35 \%$ & 13 & $65 \%$ & 20 \\
\hline \multicolumn{6}{|l|}{ zu Urologie / Urologie: } \\
\hline Operative Urologie / Urologie opératoire & 4 & $2 \%$ & 166 & $98 \%$ & 170 \\
\hline Total Schwerpunkte / Formations approfondies & 374 & $18 \%$ & $1 ' 656$ & $82 \%$ & $2 ' 030$ \\
\hline
\end{tabular}

\begin{tabular}{|c|c|c|c|c|c|}
\hline \multicolumn{6}{|l|}{ Fähigkeitsausweise / Attestations de formation complémentaire: } \\
\hline Akupunktur-TCM (ASA) / Acupuncture-MTC (ASA) & 233 & $38 \%$ & 377 & $62 \%$ & 610 \\
\hline Anthroposophisch erweiterte Medizin (VAOAS) / Médecine d'orientation anthroposophique (ASMOA) & 30 & $33 \%$ & 60 & $67 \%$ & 90 \\
\hline Delegierte Psychotherapie (FMPP) / Psychothérapie déléguée (FMPP) & 26 & $25 \%$ & 76 & $75 \%$ & 102 \\
\hline Elektroencephalographie (SGKN) / Électroencéphalographie (SSNC) & 45 & $21 \%$ & 174 & $79 \%$ & 219 \\
\hline Elektroneuromyographie (SGKN) / Électroneuromyographie (SSNC) & 49 & $21 \%$ & 183 & $79 \%$ & 232 \\
\hline $\begin{array}{l}\text { Endoskopisch Retrograde Cholangio-Pankreatographie ERCP (SGG) / Cholangio-pancréatographie } \\
\text { endoscopique rétrograde ERCP (SSG) }\end{array}$ & 2 & $4 \%$ & 53 & $96 \%$ & 55 \\
\hline Gastroskopie (SGG) / Gastroscopie (SSG) & 1 & $3 \%$ & 33 & $97 \%$ & 34 \\
\hline Homöopathie (SVHA) / Homéopathie (SSMH) & 125 & $44 \%$ & 157 & $56 \%$ & 282 \\
\hline Hüftsonographie (SGUM) / Sonographie de la hanche (SSUM) & 87 & $30 \%$ & 205 & $70 \%$ & 292 \\
\hline $\begin{array}{l}\text { Laserbehandlungen der Haut und hautnahen Schleimhäute (FMS) / Laserthérapie de la peau et des } \\
\text { muqueuses orificielles (FMS) }\end{array}$ & 81 & $21 \%$ & 312 & $79 \%$ & 393 \\
\hline Manuelle Medizin (SAMM) / Médecine manuelle (SMSMM) & 150 & $13 \%$ & 1015 & $87 \%$ & $1 ' 165$ \\
\hline Medizinische Hypnose (SMSH/GHypS) / Hypnose médicale (SMSH/SHypS) & 44 & $25 \%$ & 130 & $75 \%$ & $\overline{174}$ \\
\hline Neuraltherapie (SANTH) / Thérapie neurale (SMSTN) & 27 & $23 \%$ & 93 & $78 \%$ & 120 \\
\hline Notarzt SGNOR / Médecin d'urgence SSMUS & 139 & $26 \%$ & 391 & $74 \%$ & 530 \\
\hline Phlebologie (USGG) / Phlébologie (USSMV) & 34 & $23 \%$ & 112 & $77 \%$ & 146 \\
\hline Praxislabor (KHM) / Pratique du laboratoire au cabinet médical (CMPR) & 1691 & $21 \%$ & 6184 & $79 \%$ & $7 ' 875$ \\
\hline $\begin{array}{l}\text { Psychosomatische und Psychosoziale Medizin (APPM) / Médecine psychosomatique et } \\
\text { psychosociale de l'AMPP }\end{array}$ & 260 & $43 \%$ & 348 & $57 \%$ & 608 \\
\hline $\begin{array}{l}\text { Sachkunde f.dosisint.Untersuchungen \& therapeut.Eingriffe i.d.Angiologie (USGG) / Qual. pour les } \\
\text { ex. et traitements radiol. à fortes doses en angiologie (USSMV) }\end{array}$ & 8 & $20 \%$ & 33 & $80 \%$ & 41 \\
\hline $\begin{array}{l}\text { Sachkunde für dosisintensive Röntgenuntersuchungen in der Kardiologie (SGK) / Qualification pour } \\
\text { les ex. radiologiques à fortes doses en cardiologie (SSC) }\end{array}$ & 20 & $8 \%$ & 237 & $92 \%$ & 257 \\
\hline $\begin{array}{l}\text { Sachkunde für dosisintensives Röntgen (KHM) / Qualification pour les examens radiologiques à } \\
\text { fortes doses (CMPR) }\end{array}$ & 584 & $15 \%$ & 3325 & $85 \%$ & 3'909 \\
\hline Schwangerschaftsultraschall (SGUM) / Ultrasonographie prénatale (SSUM) & 457 & $40 \%$ & 675 & $60 \%$ & $1 ' 132$ \\
\hline Sonographie des Abdomens (SGUM) / Ultrasonographie de l'abdomen (SSUM) & 146 & $14 \%$ & 908 & $86 \%$ & 1'054 \\
\hline Sportmedizin (SGSM) / Médecine du sport (SSMS) & 28 & $9 \%$ & 267 & $91 \%$ & 295 \\
\hline Tauchmedizin (SUHMS) / Médecine de plongée (SUHMS) & 10 & $20 \%$ & 39 & $80 \%$ & 49 \\
\hline Vertrauensarzt (SGV) / Médecin-conseil (SSMC) & 37 & $17 \%$ & 180 & $83 \%$ & 217 \\
\hline Zerebrovaskuläre Krankheiten (SGKN) / Maladies cérébrovasculaires (SSNC) & 23 & $18 \%$ & 107 & $82 \%$ & 130 \\
\hline Total Fähigkeitsausweise / Attestations de formation complémentaire & 4'337 & $22 \%$ & $15^{\prime} 674$ & $78 \%$ & $20 ' 011$ \\
\hline
\end{tabular}




\section{Verleihung von eidg. Weiterbildungstiteln 2006 / Octroi de titres fédéraux de spécialiste en 2006}

\begin{tabular}{|c|c|c|c|c|c|}
\hline Facharzttitel / Titres de spécialiste & \begin{tabular}{c|c|} 
Frauen I \\
Femmes
\end{tabular} & $\begin{array}{r}\text { in } \% / \\
\text { en } \%\end{array}$ & \begin{tabular}{l|} 
Männer $/$ \\
Hommes
\end{tabular} & $\begin{array}{r}\text { in } \% / \\
\text { en } \%\end{array}$ & Total \\
\hline Allergologie und klinische Immunologie / Allergologie et immunologie clinique & 0 & $0 \%$ & 3 & $100 \%$ & \\
\hline Allgemeinmedizin / Médecine générale & 52 & $41 \%$ & 76 & $59 \%$ & 128 \\
\hline Anästhesiologie / Anesthésiologie & 16 & $38 \%$ & 26 & $62 \%$ & 42 \\
\hline Angiologie / Angiologie & 2 & $40 \%$ & 3 & $60 \%$ & 5 \\
\hline Arbeitsmedizin / Médecine du travail & 2 & $33 \%$ & 4 & $67 \%$ & 6 \\
\hline Chirurgie / Chirurgie & 7 & $19 \%$ & 29 & $81 \%$ & 36 \\
\hline Dermatologie und Venerologie / Dermatologie et vénéréologie & 7 & $35 \%$ & 13 & $65 \%$ & 20 \\
\hline Endokrinologie/Diabetologie / Endocrinologie/diabétologie & 1 & $17 \%$ & 5 & $83 \%$ & 6 \\
\hline Gastroenterologie / Gastroentérologie & 1 & $13 \%$ & 7 & $88 \%$ & 8 \\
\hline Gynäkologie und Geburtshilfe / Gynécologie et obstétrique & 10 & $56 \%$ & 8 & $44 \%$ & 18 \\
\hline Hämatologie / Hématologie & 0 & $0 \%$ & 2 & $100 \%$ & 2 \\
\hline Handchirurgie / Chirurgie de la main (kein eidg. Titel/pas de titre fédéral) & 0 & $0 \%$ & 4 & $100 \%$ & 4 \\
\hline Infektiologie / Infectiologie & 3 & $38 \%$ & 5 & $63 \%$ & 8 \\
\hline Innere Medizin / Médecine interne & 60 & $41 \%$ & 85 & $59 \%$ & 145 \\
\hline Intensivmedizin / Médecine intensive & 2 & $13 \%$ & 14 & $88 \%$ & 16 \\
\hline Kardiologie / Cardiologie & 5 & $16 \%$ & 26 & $84 \%$ & 31 \\
\hline Kiefer- und Gesichtschirurgie / Chirurgie maxillo-faciale & 0 & $0 \%$ & 2 & $100 \%$ & 2 \\
\hline Kinder- und Jugendmedizin / Pédiatrie & 54 & $67 \%$ & 27 & $33 \%$ & 81 \\
\hline $\begin{array}{l}\text { Kinder- und Jugendpsychiatrie und -psychotherapie / Psychiatrie et } \\
\text { psychothérapie d'enfants et d'adolescents }\end{array}$ & 19 & $66 \%$ & 10 & $34 \%$ & 29 \\
\hline Kinderchirurgie / Chirurgie pédiatrique & 2 & $100 \%$ & 0 & $0 \%$ & 2 \\
\hline Klinische Pharmakologie und Toxikologie / Pharmacologie et toxicologie cliniques & 1 & $100 \%$ & 0 & $0 \%$ & \\
\hline Medizinische Genetik / Génétique médicale & 1 & $50 \%$ & 1 & $50 \%$ & 2 \\
\hline Medizinische Onkologie / Oncologie médicale & 8 & $47 \%$ & 9 & $53 \%$ & 17 \\
\hline Nephrologie / Néphrologie & 5 & $63 \%$ & 3 & $38 \%$ & 8 \\
\hline Neurochirurgie / Neurochirurgie & 1 & $20 \%$ & 4 & $80 \%$ & \\
\hline Neurologie / Neurologie & 0 & $0 \%$ & 6 & $100 \%$ & 6 \\
\hline Nuklearmedizin / Médecine nucléaire & 1 & $17 \%$ & 5 & $83 \%$ & 6 \\
\hline Ophthalmologie / Ophtalmologie & 16 & $59 \%$ & 11 & $41 \%$ & 27 \\
\hline $\begin{array}{l}\text { Orthopädische Chirurgie und Traumatologie des Bewegungsapparates / } \\
\text { Chirurgie orthopédique et traumatologie de l'appareil locomoteur }\end{array}$ & 2 & $6 \%$ & 30 & $94 \%$ & 32 \\
\hline Oto-Rhino-Laryngologie / Oto-rhino-laryngologie & 4 & $36 \%$ & 7 & $64 \%$ & 11 \\
\hline Pathologie / Pathologie & 5 & $50 \%$ & 5 & $50 \%$ & 10 \\
\hline Pharmazeutische Medizin / Médecine pharmaceutique & 4 & $57 \%$ & 3 & $43 \%$ & \\
\hline Physikalische Medizin und Rehabilitation / Médecine physique et réadaptation & 3 & $43 \%$ & 4 & $57 \%$ & 7 \\
\hline $\begin{array}{l}\text { Plastische, Rekonstruktive und Ästhetische Chirurgie / Chirurgie plastique, } \\
\text { reconstructive et esthétique }\end{array}$ & 3 & $38 \%$ & 5 & $63 \%$ & \\
\hline Pneumologie / Pneumologie & 2 & $14 \%$ & 12 & $86 \%$ & 14 \\
\hline Prävention und Gesundheitswesen / Prévention et santé publique & 3 & $100 \%$ & 0 & $0 \%$ & 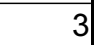 \\
\hline Psychiatrie und Psychotherapie / Psychiatrie et psychothérapie & 77 & $55 \%$ & 64 & $45 \%$ & 141 \\
\hline Radiologie / Radiologie & 8 & $27 \%$ & 22 & $73 \%$ & 30 \\
\hline Radio-Onkologie / Strahlentherapie / Radio-oncologie / radiothérapie & 2 & $33 \%$ & 4 & $67 \%$ & 6 \\
\hline Rechtsmedizin / Médecine légale & 0 & $0 \%$ & 4 & $100 \%$ & 4 \\
\hline Rheumatologie / Rhumatologie & 0 & $0 \%$ & 8 & $100 \%$ & 8 \\
\hline Tropen- und Reisemedizin / Médecine tropicale et médecine de voyages & 0 & $0 \%$ & 2 & $100 \%$ & 2 \\
\hline Urologie / Urologie & 0 & $0 \%$ & 5 & $100 \%$ & 5 \\
\hline Total Facharzttitel / Total titres de spécialiste & 389 & $41 \%$ & 563 & $59 \%$ & 952 \\
\hline Praktischer $A$ & 53 & $44 \%$ & 68 & $56 \%$ & 121 \\
\hline
\end{tabular}




\section{Verleihung von FMH-Schwerpunkten und FMH- Fähigkeitsausweisen 2006 / Octroi de titres de formations approfondies et attestation de formation complémentaire en FMH 2006}

\begin{tabular}{|c|c|c|c|c|c|}
\hline & \begin{tabular}{|c|} 
Frauen / \\
Femmes \\
\end{tabular} & \begin{tabular}{r|} 
in $\% /$ \\
en $\%$ \\
\end{tabular} & $\begin{array}{l}\text { Männer / } \\
\text { Hommes } \\
\end{array}$ & \begin{tabular}{r|} 
in $\% /$ \\
en $\%$ \\
\end{tabular} & Total \\
\hline \multicolumn{6}{|l|}{ Schwerpunkte / Formations approfondies: } \\
\hline \multicolumn{6}{|l|}{ zu Allgemeinmedizin oder Innere Medizin / Médecine générale ou médecine interne: } \\
\hline Geriatrie / gériatrie & $4 \mid$ & $31 \%$ & 9 & $69 \%$ & 13 \\
\hline \multicolumn{6}{|c|}{ zu Chirurgie oder Herz- und thorakale Gefässchirurgie / Chirurgie ou chirurgie cardiaque et vasculaire thoracique: } \\
\hline Gefässchirurgie / Chirurgie vasculaire & 0 & $0 \%$ & 5 & $100 \%$ & 5 \\
\hline Thoraxchirurgie / Chirurgie thoracique & 0 & $0 \%$ & 6 & $100 \%$ & 6 \\
\hline \multicolumn{6}{|l|}{ zu Chirurgie / Chirurgie: } \\
\hline Allgemeinchirurgie und Unfallchirurgie / Chirurgie générale et d'urgence & $2 \mid$ & $5 \%$ & 35 & $95 \%$ & 37 \\
\hline Viszeralchirurgie / Chirurgie viscérale & 3 & $7 \%$ & 40 & $93 \%$ & 43 \\
\hline \multicolumn{6}{|l|}{ zu Gynäkologie \& Geburtshilfe / Gynécologie et obstétrique: } \\
\hline Geburtshilfe und feto-maternale Medizin / Obstétrique et médecine foeto-maternelle & 1 & $50 \%$ & 1 & $50 \%$ & 2 \\
\hline $\begin{array}{l}\text { Reproduktionsmedizin und gynäkologische Endokrinologie / Médecine de la reproduction et endocrinologie } \\
\text { gynécologique }\end{array}$ & 4 & $80 \%$ & 1 & $20 \%$ & 5 \\
\hline \multicolumn{6}{|l|}{ zu Kinder- und Jugendmedizin / Pédiatrie: } \\
\hline Neoantologie / Néonatologie & 1 & $100 \%$ & 0 & $0 \%$ & \\
\hline Neuropädiatrie / Neuropédiatrie & 1 & $50 \%$ & 1 & $50 \%$ & 2 \\
\hline Pädiatrische Endokrinologie-Diabetologie / Endocrinologie-diabétologie pédiatrique & 1 & $100 \%$ & 0 & $0 \%$ & 1 \\
\hline Pädiatrische Gastroenterologie / Gastroentérologie pédiatrique & 1 & $33 \%$ & 2 & $67 \%$ & 3 \\
\hline Pädiatrische Kardiologie / Cardiologie pédiatrique & 1 1. & $50 \%$ & 1 & $50 \%$ & 2 \\
\hline Pädiatrische Onkologie-Hämatologie / Oncologie-hématologie pédiatrique & 0 & $0 \%$ & 2 & $100 \%$ & 2 \\
\hline Pädiatrische Pneumologie / Pneumologie pédiatrique & 0 & $0 \%$ & 3 & $100 \%$ & 3 \\
\hline \multicolumn{6}{|l|}{ zu Ophtalmologie / Ophtalmologie: } \\
\hline Ophthalmochirurgie / Ophtalmochirurgie & 7 & $44 \%$ & 9 & $56 \%$ & 16 \\
\hline \multicolumn{6}{|l|}{ zu Oto-Rhino-Laryngologie / Oto-rhino-laryngologie: } \\
\hline Hals- und Gesichtschirurgie / Chirurgie cervico-faciale & 2 & $25 \%$ & 6 & $75 \%$ & 8 \\
\hline Phoniatrie / Phoniatrie & 1 & $100 \%$ & 0 & $0 \%$ & 1 \\
\hline \multicolumn{6}{|l|}{ zu Pathologie / Pathologie: } \\
\hline Molekularpathologie / Pathologie moléculaire & 1 1. & $100 \%$ & 0 & $0 \%$ & 1 \\
\hline Zytopathologie / Cytopathologie & 2 & $100 \%$ & 0 & $0 \%$ & 2 \\
\hline \multicolumn{6}{|l|}{ zu Psychiatrie und Psychotherapie: } \\
\hline Alterspsychiatrie und Alterspsychotherapie / Psychiatrie et psychothérapie de la personne âgée & 3 & $30 \%$ & 7 & $70 \%$ & 10 \\
\hline \multicolumn{6}{|l|}{ zu Radiologie / Radiologie: } \\
\hline Diagnostische Neuroradiologie / Neuroradiologie diagnostique & 0 & $0 \%$ & 7 & $100 \%$ & 7 \\
\hline Pädiatrische Radiologie / Radiologie pédiatrique & 2 & $67 \%$ & 1 & $33 \%$ & 3 \\
\hline \multicolumn{6}{|l|}{ zu Urologie / Urologie: } \\
\hline Operative Urologie / Urologie opératoire & 0 & $0 \%$ & 3 & $100 \%$ & 3 \\
\hline Total Schwerpunkte / Formations approfondies & 37 & $21 \%$ & 139 & $79 \%$ & 176 \\
\hline
\end{tabular}

Fähigkeitsausweise / Attestations de formation complémentaire:

Akupunktur-TCM (ASA) / Acupuncture-MTC (ASA)

Anthroposophisch erweiterte Medizin (VAOAS) / Médecine d'orientation anthroposophique (ASMOA)

Delegierte Psychotherapie (FMPP) / Psychothérapie déléguée (FMPP)

Elektroencephalographie (SGKN) / Électroencéphalographie (SSNC)

Elektroneuromyographie (SGKN) / Électroneuromyographie (SSNC)

Endoskopisch Retrograde Cholangio-Pankreatographie ERCP (SGG) / Cholangio-pancréatographie

endoscopique rétrograde ERCP (SSG)

Gastroscopie (SSG)

Homöopathie (SVHA) / Homéopathie (SSMH)

Hüftsonographie (SGUM) / Sonographie de la hanche (SSUM)

Laserbehandlungen der Haut und hautnahen Schleimhäute (FMS) / Laserthérapie de la peau et des

muqueuses orificielles (FMS)

Manuelle Medizin (SAMM) / Médecine manuelle (SMSMM)

Medizinische Hypnose (SMSH/GHypS) / Hypnose médicale (SMSH/SHypS)

Neuraltherapie (SANTH) / Thérapie neurale (SMSTN)

Notarzt SGNOR / Médecin d'urgence SSMUS

Phlebologie (USGG) / Phlébologie (USSMV)

Praxislabor (KHM) / Pratique du laboratoire au cabinet médical (CMPR)

Psychosomatische und Psychosoziale Medizin (APPM) / Médecine psychosomatique et psychosociale de I'AMPP

Sachkunde f.dosisint.Untersuchungen \& therapeut.Eingriffe i.d.Angiologie (USGG) / Qual. pour les ex. et traitements radiol. à fortes doses en angiologie (USSMV)

Sachkunde für dosisintensive Röntgenuntersuchungen in der Kardiologie (SGK) / Qualification pour les ex. radiologiques à fortes doses en cardiologie (SSC)

Sachkunde für dosisintensives Röntgen (KHM) / Qualification pour les examens radiologiques à fortes doses (CMPR)

Schwangerschaftsultraschall (SGUM) / Ultrasonographie prénatale (SSUM)

Sonographie des Abdomens (SGUM) / Ultrasonographie de l'abdomen (SSUM)

Sportmedizin (SGSM) / Médecine du sport (SSMS)

Vertrauensarzt (SGV) / Médecin-conseil (SSMC)

Zerebrovaskuläre Krankheiten (SGKN) / Maladies cérébrovasculaires (SSNC)

Total Fähigkeitsausweise / Attestations de formation complémentaire

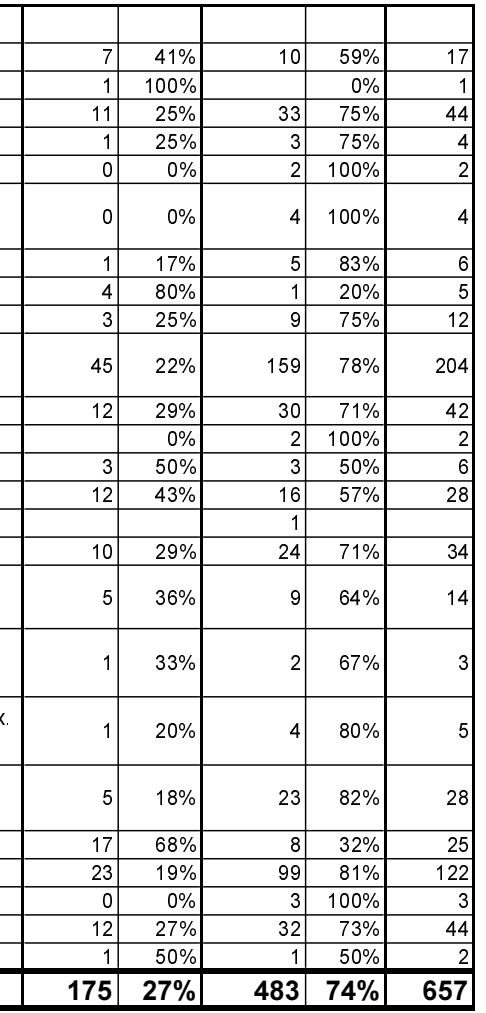

(c) FMH-Generalsekretariat 2007, Stand per 31.12.2006 


\section{Altersstruktur 2006 / Structure d' âge en 2006}
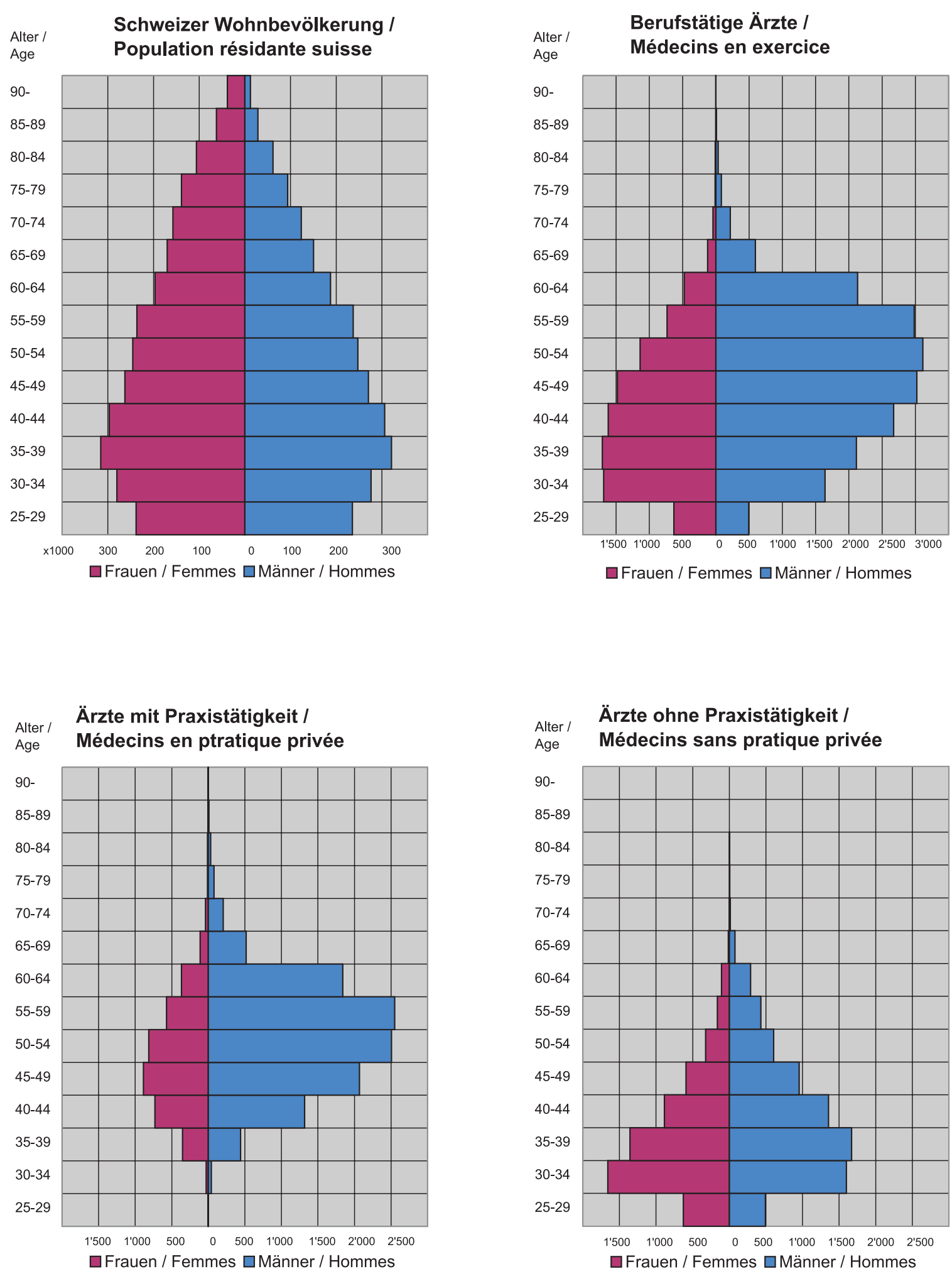


\section{Vergleichszahlen 2002-2006 / Comparaison 2002-2006}

\begin{tabular}{|c|c|c|c|c|c|}
\hline Ärzte in der Schweiz I Médecins en Suisse & 2006 & 2005 & 2004 & 2003 & 2002 \\
\hline Total berufstätige Ärzte / Médecins en exercice & $28 ' 812$ & $28{ }^{\prime} 251$ & $27^{\prime} 742$ & $27^{\prime} 268$ & $25^{\prime} 921$ \\
\hline Frauen / Femmes & 9'682 & 9'291 & $8^{\prime} 934$ & $8^{\prime} 659$ & $7 ' 946$ \\
\hline in \% aller berufstätigen Ärzte / en \% de tous les médecins en exercice & $33.6 \%$ & $32.9 \%$ & $32.2 \%$ & $31.8 \%$ & $30.7 \%$ \\
\hline Männer / Hommes & $19^{\prime} 130$ & $18^{\prime} 960$ & $18^{\prime} 808$ & $18^{\prime} 609$ & $17^{\prime} 975$ \\
\hline in \% aller berufstätigen Ärzte / en \% de tous les médecins en exercice & $66.4 \%$ & $67.1 \%$ & $67.8 \%$ & $68.2 \%$ & $69.3 \%$ \\
\hline Ärzte mit Praxistätigkeit / Médecins en pratique privée & $15^{\prime} 532$ & $15^{\prime} 313$ & $15^{\prime} 199$ & $14 ' 879$ & $14^{\prime} 408$ \\
\hline in \% aller berufstätigen Ärzte / en \% de tous les médecins en exercice & $53.9 \%$ & $54.2 \%$ & $54.8 \%$ & $54.6 \%$ & $55.6 \%$ \\
\hline \multicolumn{6}{|c|}{ Aufteilung der Ärzte mit Praxistätigkeit / Répartition des médecins en pratique privée: } \\
\hline Fachärzte für Allgemeinmedizin / Spécialistes en médecine générale & $22.5 \%$ & $22.7 \%$ & $22.6 \%$ & $22.3 \%$ & $20.5 \%$ \\
\hline Übrige Fachärzte / Autres spécialistes & $70.4 \%$ & $69.7 \%$ & $69.3 \%$ & $68.9 \%$ & $66.2 \%$ \\
\hline Ärzte ohne Facharzttitel / Médecins sans titre de spécialiste & $7.1 \%$ & $7.6 \%$ & $8.1 \%$ & $8.8 \%$ & $13.4 \%$ \\
\hline - davon Praktischer Arzt / Médecin praticien & $34.5 \%$ & $34.1 \%$ & $31.2 \%$ & $26.3 \%$ & $16.6 \%$ \\
\hline Ärzte ohne Praxistätigkeit / Médecins sans pratique privée & $13^{\prime} 280$ & $12^{\prime} 938$ & $12 ' 543$ & $12 ' 389$ & $11^{\prime} 513$ \\
\hline in \% aller berufstätigen Ärzte / en \% de tous les médecins en exercice & $46.1 \%$ & $45.8 \%$ & $45.2 \%$ & $45.4 \%$ & $44.4 \%$ \\
\hline \multicolumn{6}{|c|}{ Aufteilung der Ärzte ohne Praxistätigkeit / Répartition des médecins sans pratique privée: } \\
\hline Fachärzte für Allgemeinmedizin / Spécialistes en médecine générale & $3.2 \%$ & $2.8 \%$ & $2.9 \%$ & $2.8 \%$ & $2.2 \%$ \\
\hline Übrige Fachärzte / Autres spécialistes & $42.5 \%$ & $40.4 \%$ & $37.6 \%$ & $35.5 \%$ & $32.7 \%$ \\
\hline Ärzte ohne Facharzttitel / Médecins sans titre de spécialiste & $54.2 \%$ & $56.7 \%$ & $59.5 \%$ & $61.7 \%$ & $65.1 \%$ \\
\hline - davon Praktischer Arzt / Médecin praticien & $5.3 \%$ & $4.8 \%$ & $4.9 \%$ & $5.2 \%$ & $5.0 \%$ \\
\hline Total berufstätige Ärzte / Médecins en exercice & $28 ' 812$ & $28 ' 251$ & $27^{\prime} 742$ & $27 ' 268$ & $25^{\prime} 921$ \\
\hline \multicolumn{6}{|l|}{ Aufteilung der berufstätigen Ärzte / Répartition des médecins en exercice: } \\
\hline Fachärzte für Allgemeinmedizin / Spécialistes en médecine générale & $13.6 \%$ & $13.6 \%$ & $13.7 \%$ & $13.4 \%$ & $12.4 \%$ \\
\hline Übrige Fachärzte / Autres spécialistes & $57.6 \%$ & $56.3 \%$ & $55.0 \%$ & $53.7 \%$ & $51.3 \%$ \\
\hline Ärzte ohne Facharzttitel / Médecins sans titre de spécialiste & $28.8 \%$ & $30.1 \%$ & $31.3 \%$ & $32.9 \%$ & $36.3 \%$ \\
\hline \multirow{2}{*}{ - davon Praktischer Arzt / Médecin praticien } & $9.2 \%$ & $8.8 \%$ & $8.6 \%$ & $8.3 \%$ & $7.4 \%$ \\
\hline & $100.0 \%$ & $100.0 \%$ & $100.0 \%$ & $100.0 \%$ & $100.0 \%$ \\
\hline \multicolumn{6}{|l|}{ Anzahl Einwohner pro / Nombre d'habitants par: } \\
\hline Arzt mit Praxistätigkeit / Médecin en pratique privée & 487 & 490 & 491 & 498 & 510 \\
\hline Berufstätigen Arzt / Médecin en exercice & 262 & 266 & 269 & 272 & 284 \\
\hline \multicolumn{6}{|c|}{ Durchschnittsalter der berufstätigen Ärzte / Age moyen des médecins en exercice: } \\
\hline Berufstätige Ärzte / Médecins en exercice & 47 & 46 & 46 & 45 & 45 \\
\hline Frauen / Femmes & 43 & 43 & 42 & 42 & 42 \\
\hline Männer / Hommes & 49 & 48 & 48 & 47 & 47 \\
\hline Ärzte mit Praxistätigkeit / Médecins en pratique privée & 52 & 52 & 52 & 51 & 51 \\
\hline Ärzte ohne Praxistätigkeit / Médecins sans pratique privée & 40 & 40 & 39 & 39 & 38 \\
\hline Durchschnittliches Sterbealter / L'âge moven des décès & 77 & 75 & 76 & 75 & 75 \\
\hline
\end{tabular}




\section{FMH-Mitgliederstatistik 2006 I}

\section{Statistique des membres FMH 2006}

\section{A) Basisorganisationen / Organisations de base}

Mitgliederbestand per 1.1.2007 / Effectif des membres au 1.1.2007

\begin{tabular}{|c|c|c|c|c|c|c|}
\hline & Kantonale Ärztegesellschaften / Sociétés cantonales & $\begin{array}{l}\text { Frauen } / \\
\text { femmes }\end{array}$ & $\begin{array}{r}\text { in } \% / \\
\text { en } \%\end{array}$ & $\begin{array}{l}\text { Männer / } \\
\text { hommes }\end{array}$ & $\begin{array}{r}\text { in } \% / \\
\text { en } \%\end{array}$ & Total \\
\hline AG & Aargauischer Ärzteverband & 249 & $23 \%$ & 849 & $77 \%$ & $1 ' 098$ \\
\hline Al/AR & Appenzellische Ärztegesellschaft & 27 & $21 \%$ & 102 & $79 \%$ & 129 \\
\hline BL & Ärztegesellschaft Basel-Land & 180 & $27 \%$ & 478 & $73 \%$ & 658 \\
\hline BS & Medizinische Gesellschaft Basel & 351 & $29 \%$ & 864 & $71 \%$ & 1'215 \\
\hline BE & Ärztegesellschaft des Kantons Bern & 599 & $22 \%$ & 2'102 & $78 \%$ & 2'701 \\
\hline FR & Société de médecine du canton de Fribourg & 106 & $23 \%$ & 365 & $77 \%$ & 471 \\
\hline GE & Association des médecins du canton de Genève & 642 & $31 \%$ & $1^{\prime} 412$ & $69 \%$ & $2 ' 054$ \\
\hline GL & Ärztegesellschaft des Kantons Glarus & 15 & $18 \%$ & 69 & $82 \%$ & 84 \\
\hline GR & Bündner Ärzteverein & 95 & $19 \%$ & 396 & $81 \%$ & 491 \\
\hline JU & Société médicale du canton du Jura & 26 & $19 \%$ & 114 & $81 \%$ & 140 \\
\hline LU & Ärztegesellschaft des Kantons Luzern & 149 & $19 \%$ & 633 & $81 \%$ & 782 \\
\hline NE & Société neuchâteloise de médecine & 106 & $23 \%$ & 351 & $77 \%$ & 457 \\
\hline SG & Ärztegesellschaft des Kantons St. Gallen & 203 & $19 \%$ & 840 & $81 \%$ & $1 ' 043$ \\
\hline SH & Kantonale Ärztegesellschaft Schaffhausen & 41 & $22 \%$ & 144 & $78 \%$ & 185 \\
\hline SZ & Ärztegesellschaft des Kantons Schwyz & 48 & $24 \%$ & 156 & $76 \%$ & 204 \\
\hline so & Gesellschaft der Ärztinnen und Ärzte des Kantons Solothurn & 114 & $22 \%$ & 396 & $78 \%$ & 510 \\
\hline TI & Ordine dei medici del cantone Ticino & 210 & $22 \%$ & 760 & $78 \%$ & 970 \\
\hline TG & Thurgauische Ärztegesellschaft & 85 & $20 \%$ & 331 & $80 \%$ & 416 \\
\hline NWIOW & Unterwaldner Ärztegesellschaft & 18 & $15 \%$ & 99 & $85 \%$ & 117 \\
\hline UR & Verband der Urner Ärzte & 7 & $13 \%$ & 46 & $87 \%$ & 53 \\
\hline VD & Société vaudoise de médecine & 646 & $28 \%$ & $1^{\prime} 686$ & $72 \%$ & $2^{\prime} 332$ \\
\hline VS & Société médicale du Valais / Walliser Ärzteverband & 123 & $20 \%$ & 498 & $80 \%$ & 621 \\
\hline ZG & Ärztegesellschaft des Kantons Zug & 54 & $22 \%$ & 192 & $78 \%$ & 246 \\
\hline ZH & Ärztegesellschaft des Kantons Zürich & $1 ' 244$ & $29 \%$ & 2'982 & $71 \%$ & 4'226 \\
\hline & $\begin{array}{l}\text { Total kantonale Ärztegesellschaften I } \\
\text { Total sociétés cantonales }\end{array}$ & $5^{\prime} 338$ & $25 \%$ & $15 ' 865$ & $75 \%$ & $21 ' 203$ \\
\hline $\begin{array}{l}\text { VSAO I } \\
\text { ASMAC }\end{array}$ & $\begin{array}{l}\text { Verband Schweizerischer Assistenz- und Oberärzte/-innen / } \\
\text { Association suisse des médecins-assistants et chefs de } \\
\text { clinique }\end{array}$ & $5^{\prime} 101$ & $48 \%$ & $5^{\prime} 583$ & $52 \%$ & $10^{\prime} 684$ \\
\hline AMINE & Ass. des médecins d'institutions neuchâteloises & 12 & $57 \%$ & 9 & $43 \%$ & 21 \\
\hline \multirow[t]{2}{*}{$\begin{array}{l}\text { VLSS / } \\
\text { AMDHS }\end{array}$} & $\begin{array}{l}\text { Verein der Leitenden Spitalärzte der Schweiz / Association } \\
\text { des médecins dirigeants d'hôpitaux de Suisse }\end{array}$ & 105 & $10 \%$ & 927 & $90 \%$ & $1 ' 032$ \\
\hline & $\begin{array}{l}\text { Gesamttotal Basisorganisationen I } \\
\text { Total des organisations de base }\end{array}$ & $10 ' 556$ & $32 \%$ & $22 ' 384$ & $68 \%$ & $32 ' 940$ \\
\hline
\end{tabular}




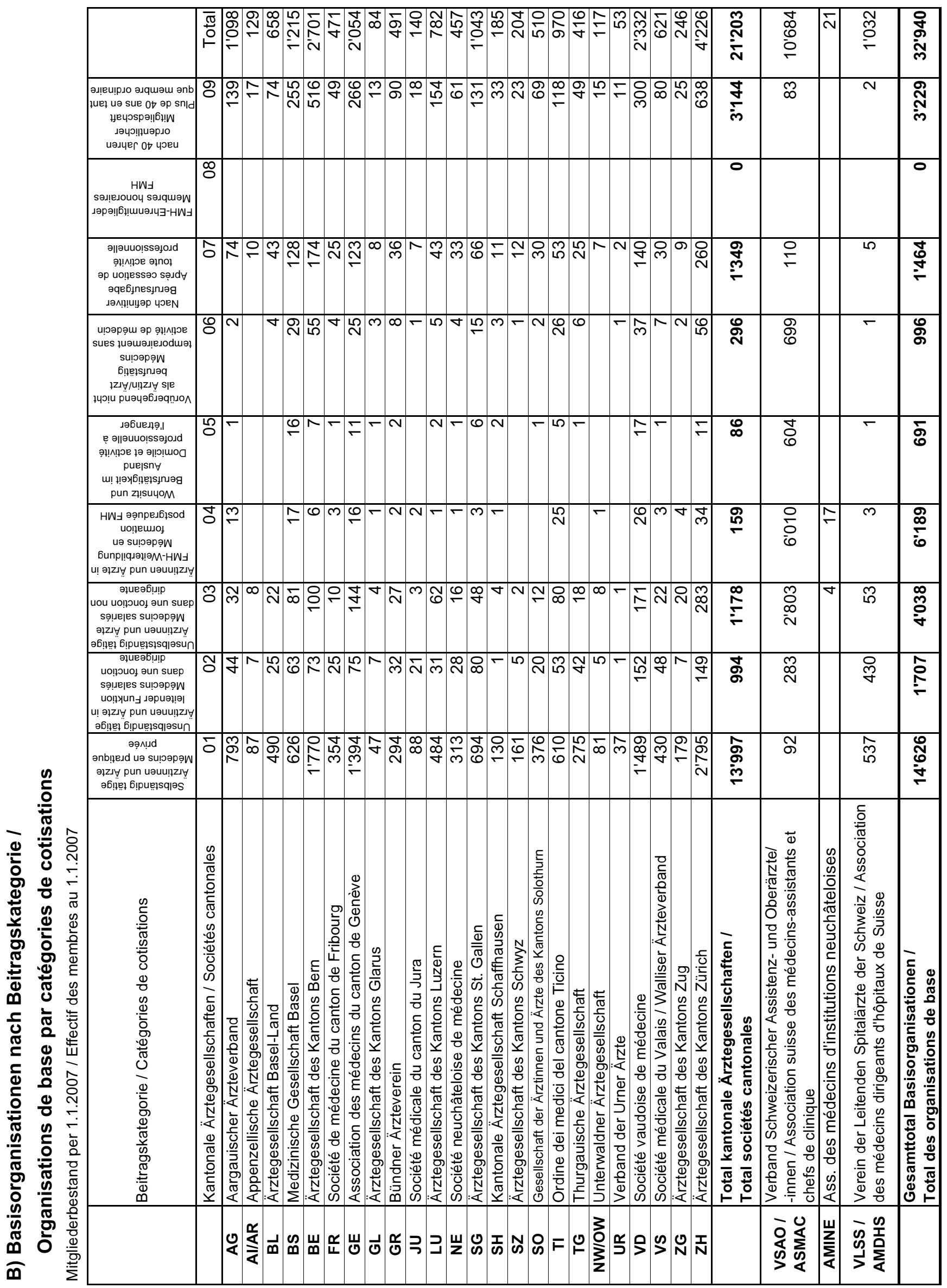




\section{C) Fachorganisationen / Organisations spécialisées}

Mitgliederbestand per 1.1.2007 / Effectif des membres au 1.1.2007

\begin{tabular}{|c|c|}
\hline Fachgesellschaften / sociétés de discipline médicale & Total \\
\hline Allergologie \& klin. Immunologie / Allergologie et immunologie clinique & 170 \\
\hline Allgemeinmedizin / Médecine générale & $3 ' 329$ \\
\hline Anästhesiologie / Anesthésiologie & 676 \\
\hline Angiologie / Angiologie & 131 \\
\hline Arbeitsmedizin / Médecine du travail & 86 \\
\hline Chirurgie / Chirurgie & $1 ' 033$ \\
\hline Dermatologie und Vernerologie / Dermatologie et vénéréologie & 378 \\
\hline Endokrinologie und Diabetologie / Endocrinologie-diabétologie & 151 \\
\hline Gastroenterologie / Gastroentérologie & 253 \\
\hline Gynäkologie und Geburtshilfe / Gynécologie et obstétrique & 921 \\
\hline Hämatologie / Hématologie & 115 \\
\hline Herz- und thorakale Gefässchirurgie / Chirurgie cardiaque et vasculaire thoracique & 80 \\
\hline Infektiologie / Infectiologie & 112 \\
\hline Innere Medizin / Médecine interne & $3 ' 530$ \\
\hline Intensivmedizin / Médecine intensive & 277 \\
\hline Kardiologie / Cardiologie & 457 \\
\hline Kiefer- und Gesichtschirurgie / Chirurgie maxillo-faciale & 62 \\
\hline Kinder- und Jugendmedizin / Pédiatrie & $1 ' 225$ \\
\hline Kinder- und Jugendpsychiatrie und -psychotherapie / Psychiatrie et psychothérapie d'enfants et d'adolescents & 472 \\
\hline Kinderchirurgie / Chirurgie pédiatrique & 60 \\
\hline Klinische Pharmakologie und Toxikologie / Pharmacologie clinique et toxicologie & 33 \\
\hline Medizinische Genetik / Génétique médicale & 17 \\
\hline Medizinische Onkologie / Oncologie médicale & 199 \\
\hline Nephrologie / Néphrologie & 139 \\
\hline Neurochirurgie / Neurochirurgie & 81 \\
\hline Neurologie / Neurologie & 286 \\
\hline Nuklearmedizin / Médecine nucléaire & 53 \\
\hline Ophthalmologie / Ophthalmologie & 803 \\
\hline Orthopädische Chirurgie / Chirurgie orthopédique & 559 \\
\hline Oto-Rhino-Laryngologie / Oto-rhino-laryngologie & 409 \\
\hline Pathologie / Pathologie & 190 \\
\hline Pharmazeutische Medizin / Médecine pharmaceutique & 54 \\
\hline Physikalische Medizin und Rehabilitation / Médecine physique et réadaptation & 243 \\
\hline Plastische, Rekonstruktive und Ästhetische Chirurgie / Chirurgie plastique, reconstructive et esthétique & $1 ' 117$ \\
\hline Pneumologie / Pneumologie & 223 \\
\hline Prävention und Gesundheitswesen / Prévention et santé publique & 63 \\
\hline Psychiatrie und Psychotherapie / Psychiatrie et psychothérapie & $1 ' 581$ \\
\hline Radiologie / Radiologie & 495 \\
\hline Radio-Onkologie/Strahlentherapie / Radio-oncologie/radiothérapie & 47 \\
\hline Rechtsmedizin / Médecine légale & 26 \\
\hline Rheumatologie / Rhumatologie & 412 \\
\hline Tropen- und Reisemedizin / Médecine tropicale et médecine des voyages & 52 \\
\hline Urologie / Urologie & 179 \\
\hline Total Fachgesellschaften / Total sociétés de discipline médicale & $20 ' 779$ \\
\hline Verein der Leitenden Spitalärzte der Schweiz / Association des médecins dirigeants d'hôpitaux de Suisse & $1 ' 032$ \\
\hline Gesamttotal Fachorganisationen / Total organisations spécialisées & $21 ' 811$ \\
\hline
\end{tabular}

